\title{
Galaxy Morphologies in the Hubble Ultra Deep Field: Dominance of Linear Structures at the Detection Limit
}

\author{
Debra Meloy Elmegreen \\ Vassar College, Dept. of Physics \& Astronomy, Box 745, Poughkeepsie, NY 12604; e-mail: \\ elmegreen@vassar.edu \\ Bruce G. Elmegreen \\ IBM Research Division, T.J. Watson Research Center, P.O. Box 218, Yorktown Heights, \\ NY 10598,USA; e-mail: bge@watson.ibm.com \\ Douglas S. Rubin \\ Wesleyan University, Dept. of Astronomy, Middletown, CT; e-mail:rubin@wesleyan.edu \\ Meredith A. Schaffer \\ Vassar College, Dept. of Physics \& Astronomy, Box 745, Poughkeepsie, NY 12604; \\ e-mail:meschaffer@vassar.edu
}

\begin{abstract}
Galaxies in the Hubble Ultra Deep Field (UDF) larger than 10 pixels (0.3 arcsec) have been classified according to morphology and their photometric properties are presented. There are 269 spirals, 100 ellipticals, 114 chains, 126 doubleclump, 97 tadpole, and 178 clump-cluster galaxies. We also catalogued 30 B-band and $13 \mathrm{~V}$-band drop-outs and calculated their star formation rates. Chains, doubles, and tadpoles dominate the other types at faint magnitudes. The fraction of obvious bars among spirals is $\sim 10 \%$, a factor of $2-3$ lower than in other deep surveys. The distribution function of axial ratios for elliptical galaxies is similar to that seen locally, suggesting that ellipticals relaxed quickly to a standardized shape. The distribution of axial ratios for spiral galaxies is significantly different than locally, having a clear peak at $\sim 0.55$ instead of a nearly flat distribution. The fall-off at small axial ratio occurs at a higher value than locally, indicating thicker disks by a factor of $\sim 2$. The fall-off at high axial ratio could be from intrinsic triaxial shapes or selection effects. Inclined disks should be more highly sampled than face-on disks near the surface brightness limit of a survey. Simple models and data distributions demonstrate these effects. The decreased numbers of obvious spiral galaxies at high redshifts could be partly the result of surface brightness selection.
\end{abstract}


Subject headings: galaxies: high-redshift — galaxies: evolution — galaxies: formation - galaxies: structure

\section{Introduction}

Galaxies with unusual morphologies appear at high redshift in the Hubble Deep Fields North (Williams et al. 1996) and South (Volonteri, Saracco, \& Chincarini 2000), the Hawaiian Deep Field (Cowie, Hu, \& Songaila 1995), the Tadpole Galaxy field (Tran et al. 2003), the GOODS field (Giavalisco et al. 2004) and the Hubble Ultra Deep Field (HDF; Beckwith et al. 2005). Some are recognizable as elliptical and spiral galaxies, but many at $z \geq 1.5$ have more clumpy and irregular structures (e.g., Abraham et al. 1996a,b; Conselice 2004). Chain galaxies, for example, are nearly straight alignments of a half-dozen clumps (Cowie, Hu, \& Songaila 1995; van den Bergh et al. 1996; Elmegreen, Elmegreen \& Sheets 2004, hereafter Paper I). Tadpole galaxies are curved thin structures with a big clump near one end (van den Bergh et al. 1996; Paper I; Straughn et al. 2004). Luminous diffuse objects are oval distributions of clumpy emission resembling disks (Reshetnikov, Dettmar, \& Combes 2003; Conselice et al. 2004); a subcategory of these, having no bulges or exponential disk light profiles, has been called clump-clusters (Elmegreen, Elmegreen, \& Hirst 2004a, hereafter Paper II). Double-clump galaxies have two big clumps. Most of these types are not present in the modern Universe although some probably evolve into normal Hubble types. Characteristic of all the irregular types are enormous clumps of star formation, $\sim 100$ times more massive the the largest star complexes in today's spiral galaxies, as well as thick disks and extreme asymmetries that are most likely the result of interactions and merging.

Here we present morphological data on 884 galaxies in the Hubble Ultra Deep Field (UDF). Magnitudes, colors, and axial ratios of the galaxies are studied.

\section{Data}

UDF images were obtained with the Hubble Advanced Camera for Surveys (ACS) by Beckwith et al. (2005) and are available on the Space Telescope Science Institute (STScI) archive. The images are $10500 \times 10500$ pixels with a scale of 0.03 arcsec per px (315 arcsec x 315 arcsec) in 4 filters: F435W (B band, hereafter $\mathrm{B}_{435} ; 134880 \mathrm{~s}$ exposure), F606W (V band, $\mathrm{V}_{606} ; 135320 \mathrm{~s}$ ), F775W (i band, i $775 ; 347110 \mathrm{~s}$ ), and F850LP (z band, z850; $346620 \mathrm{~s}$ ).

We used the $i_{775}$ image, the deepest of the four images, to search by eye to identify and study galaxies that have major axes larger than 10 pixels (corresponding to 0.3 arcsec). 
The whole UDF field was subdivided into 50 fields of 800x800 pixels for identification of objects. Each of these subfields was further subdivided and displayed in IRAF in 100x100 pixel increments, which were scanned by eye to search for objects larger than 10 pixels (confirmed by measuring with contour plots out to the $2 \sigma$ limit). Our full sample includes 884 galaxies. Morphological classifications were made based on the $i_{775}$ images and aided by contour plots and radial profiles. The galaxies were divided into 6 categories: chain (114 total), clump-cluster (178), double (126), tadpole (97), spiral (269), and elliptical (100). Figure 1 shows 8 examples of each type; the lines correspond to 0.5 arcsec.

Galaxy morphology can vary with wavelength, so we viewed many of the catalogued objects at other ACS passbands and with NICMOS (Thompson et al. 2005). Generally the morphological classification does not change significantly with wavelength (e.g., Dickinson 2000) because it is based on only the most fundamental galaxy characteristics, such as elongation and number of giant clumps. Also, the NICMOS images have a factor of 3 lower resolution, so they do not reveal the same fine structure as the other images.

The distinguishing characteristics of the main types we classified are:

- Chain: linear objects dominated by several giant clumps and having no exponential light profiles or central red bulges.

- Clump Cluster: oval or circular objects resembling chain galaxies in their dominance by several giant clumps, and having no exponential profiles or bulges.

- Double-clump: systems dominated by two similar clumps with no exponential profile or bulge.

- Tadpole: systems dominated by a single clump that is off-center from, or at the end of, a more diffuse linear emission.

- Spiral: galaxies with exponential-like disks, evident spiral structure if they have lowinclination, and usually a bulge or a nucleus. Edge-on spirals have relatively flat emission from a midplane, and often extended emission perpendicular to the midplane, as well as a bulge.

- Elliptical: centrally concentrated oval galaxies with no obvious spiral structure.

Chain galaxies were first recognized by Cowie et al. (1995) using the same definition as that here. Tadpole galaxies were defined by van den Bergh et al. (1996) and examples from the UDF were discussed by Straughn et al. (2004). Tadpole galaxies with short tails were classified as "comma" type in the morphology review by van den Bergh (2002). van 
den Bergh et al. (1996) also noted objects like clump-clusters and called them proto-spirals. Conselice et al. (2004) called these clump-dominated young disk galaxies "luminous diffuse objects," although some of their sample included galaxies with bulges and exponential-like profiles, unlike the clump-clusters here. Binary galaxies, like our doubles, were noted by van den Bergh (2002). Other classifications of galaxies in deep Hubble images, based on the Hubble classification or DDO systems, were made by Brinchmann et al. (1998), van den Bergh et al. (2000), and others, who generally noted that an increasing fraction of distant objects fall outside these conventional morphologies.

Distinctions between morphological types do not necessarily imply there are significant physical differences. For example, double-clump galaxies may be smaller versions of chain galaxies, and the single-clump tadpoles may be smaller yet. The origins of these types are not known. Also, chain galaxies and clump clusters may be the same type of object viewed at different orientations (Dalcanton \& Schectman 1996; O’Neil et al. 2000; Paper II).

Spiral galaxies are extremely varied. Many look highly disturbed, as if by interactions with smaller companions. Figure 2 shows four more examples of spirals. The galaxy on the left is very asymmetric and has a giant star formation region along the bottom arm. The galaxy on the right is dominated by two round clumps and an extended bright arm ("the cigar smoker").

Images of 10 different clump clusters from the UDF are in Elmegreen \& Elmegreen (2005, hereafter Paper III), where their clump properties were measured. Images of 8 different UDF ellipticals are in Elmegreen, Elmegreen, \& Ferguson (2005, hereafter Paper IV), which concentrated on those having giant blue clumps in their centers (one of the ellipticals shown in Fig. 1, UDF 4389, has blue clumps in its core, but they cannot be seen in this image).

Ellipse fits were done on all of the spirals using IRAF to search for radial variations in position angle and ellipticity. Bars have a characteristic signature on such a plot as a result of the twisting inner isophotes. The images were also viewed directly to look for bars. In general, the most highly inclined galaxies do not readily show bars even if they are present, and the short type of bar that is commonly found in local late-type galaxies could not be resolved. Nevertheless, we found 26 barred spirals out of 269 spiral galaxies, a bar fraction of $\sim 10 \%$. This is a factor of $\sim 2$ smaller than in the Tadpole field, considering even the inclination effects (Elmegreen, Elmegreen, \& Hirst 2004b, hereafter Paper V), and it is a factor of $\sim 3$ smaller than in the GOODS field (Jogee et al. 2004). The Tadpole field contained 43 barred galaxies in a sample of 186 spirals and showed clear correlations between apparent bar fraction and both inclination and galaxy angular size. The GOODS field contained 80 bars out of 258 nearly face-on spirals. Both previous studies used radial profiles of ellipticity and position angle to identify bars, as we do here. Deeper fields like 
the UDF might be expected to show fewer bars as physical resolution and surface brightness dimming become more of a problem at high redshift. Bar destruction by severe interactions (Athanassoula \& Bosma 2003) could play a role at high $z$ too. A more thorough study of the UDF bars will be addressed in another paper. Early searches for barred galaxies found relatively few examples (e.g., van den Bergh et al. 2002), probably because of the lower angular resolution of the WFPC2 camera. Sheth et al. (2003) was the first to suggest that the relative numbers of bars might be the same as that found locally.

Two examples of barred spirals in the UDF are shown in Figure 3, along with the radial profiles of ellipticity and position angle. They are the galaxies UDF 1971 (left) and UDF 9341. The bars and spirals are more irregular than in local galaxies, suggesting chaotic stellar orbits and poorly defined resonances. The bars could therefore be young. Star formation is enhanced in many small clusters near the top end of the bar in UGC 1971.

The distribution functions of the colors and magnitudes of the clumps that have been measured in chains, clump-clusters, doubles, and tadpoles, are all about the same (Paper I). Similar also are the colors and magnitudes of clumps in the cores of 30 ellipticals in the UDF (Paper IV). These colors and magnitudes give clump masses in the range from $10^{6} \mathrm{M}_{\odot}$ for the smallest ellipticals (Paper IV) to $10^{9} \mathrm{M}_{\odot}$ for the best studied clump-clusters (Paper III), based stellar population models from Bruzual \& Charlot (2003). The clumps are clearly smaller and bluer than the bulges of spiral galaxies (Paper I). The irregular morphology of the first four types suggests a history dominated by galaxy interactions, clump accretions, and large-scale instabilities leading to star formation. The highly clumpy structure of the elliptical galaxies suggests a similar history of clump accretions.

Most of the galaxies in this catalog are also listed in the UDF catalog without morphological classifications (Beckwith et al. 2005, listed on the STScI website ${ }^{1}$ ). Some of the galaxies here are not listed in the UDF catalog because they appear near bright stars or near the edge of the field of view. The parameters used for SExtractor were chosen to optimize different aspects of the UDF than our morphological system, so there is not a one-to-one correspondence. Our catalog has 9 chain galaxies, 14 clump-cluster galaxies, 10 double galaxies, and 1 tadpole galaxy with multiple UDF numbers, and there are 12 chains, 16 clump-clusters, 11 doubles, 8 tadpoles, 28 spirals and 6 ellipticals which are not in the UDF catalog at all. One object, UDF 5898, is two separate spiral galaxies which SExtractor called one, using the midpoint as a position; we call these UDF 5898A and UDF 5898B. The unlisted and multiply-listed galaxies amount to 154 objects, or $17 \%$ of the total sample.

The integrated AB magnitudes were measured in all four filters from counts within

\footnotetext{
${ }^{1}$ http://archive.stsci.edu/pub/hlsp/udf/acs-wfc/h_udf_wfc_VI_I_cat.txt
} 
a rectangular box whose edges were defined by the $i_{775}$ isophotal contours $2 \sigma$ above the sky noise (corresponding to a surface brightness of $26.0 \mathrm{mag} \operatorname{arcsec}^{-2}$ ). The integrated magnitudes depend only weakly on the choice of outer isophotal contour: 10-pixel extensions changed the magnitudes of isolated objects by less than 0.1 mag. The integrated $i_{775}-z_{850}$ colors of the galaxies range from -0.7 to 1.3 . The integrated $\mathrm{i}_{775}$ magnitudes range from 18.5 to $29 \mathrm{mag}$, with surface brightnesses from 22.5 to $27 \mathrm{mag} \operatorname{arcsec}^{-2}$. The details of some clump properties are given in separate studies of different subsamples (Papers III, IV).

Tables 1-6 list the galaxies by morphological type. In the first part of each table, the galaxies with a single UDF number are listed. In the second part, galaxies with multiple UDF numbers are given along with the combined average surface brightnesses in $i_{775}, \mu_{\text {I }}$, the apparent magnitudes, $i_{775}$, and the three colors. In the third part, galaxies with no UDF numbers are given along with the coordinates of their centers and other properties. We do not repeat the colors and magnitudes of the single sources because these measurements are in the UDF catalog; they are the same as what we measured independently to within 0.1 mag (which is the same as the measuring error for UDF magnitudes). Barred galaxies are indicated with an asterisk in the spiral list.

\section{Photometric Results}

The average color and magnitude values for each galaxy type are shown in Figure 4. This plot has more observational relevance than physical because the galaxies are a mixture of redshifts and $\mathrm{k}$-corrections. The average $i_{775}$ surface brightness is fairly constant at $\sim 24.8 \mathrm{mag} \operatorname{arcsec}^{-2}$ for all types except ellipticals, which are about 0.5 mag brighter. This constancy is likely the result of the surface brightness limit of the survey ( $2 \sigma$ is 26.0 mag $\operatorname{arcsec}^{-2}$ ), with the averages being larger than this limit because of central light concentration. The average $i_{775}$ integrated magnitude is about 26.3 for chains, doubles, and tadpoles, 24.5 for spirals, 24.2 for ellipticals, and 25.2 for clump clusters. The ellipticals have the reddest colors in all indices.

The surface brightness distribution of all the galaxies peaks at $\sim 25 \mathrm{mag} \operatorname{arcsec}^{-2}$, rapidly declines beyond the $2 \sigma$ limit of $26.0 \mathrm{mag} \operatorname{arcsec}^{-2}$, and is virtually null beyond the $3 \sigma$ limit of $26.6 \mathrm{mag} \operatorname{arcsec}^{-2}$ (see also Fig. 11 below).

Figure 5 plots $\mathrm{B}_{435}-\mathrm{V}_{606}$ versus $i_{775}-z_{850}$ for all galaxies in our sample, divided according to morphology. Note the Lyman Break galaxies of various types, which have extremely red $\mathrm{B}_{435}-\mathrm{V}_{606}$ colors. Except for these, the color distributions are similar with a large number

of galaxies at $\mathrm{B}_{435}-\mathrm{V}_{606} \sim 0$ and a second diagonal branch going up to $\mathrm{B}_{435}-\mathrm{V}_{606} \sim 2$. The 
curves superposed on the data are Bruzual \& Charlot (2003) evolution models that assume star formation began at $z=6$ for all cases and then decayed exponentially over time with e-folding times of $0.01 \mathrm{~Gy}, 0.03 \mathrm{~Gy}, 0.1 \mathrm{~Gy}, 0.3 \mathrm{~Gy}, 1 \mathrm{~Gy}, 3 \mathrm{~Gy}$, and infinity. Each curve traces the sequence of decay times with the longest decay times corresponding to the bluest colors. The different curves are for different galaxy redshifts, from 0 to 4 in steps of 0.5 (see color coding in figure caption). The models are described more in Paper III.

The distribution of colors reveals several things about these galaxies. The chains, clumpclusters, doubles, and tadpoles have similar color distributions, and the spirals have about the same distribution too, but the spirals do not have the very red $\mathrm{B}_{435}-\mathrm{V}_{606}$ and $\mathrm{i}_{775}-\mathrm{Z}_{850}$ components that are present in the more irregular systems. These red points correspond to the highest redshifts, $z \sim 4$, so the difference in distribution suggests the spirals do not generally go to as high a redshift as the more irregular and clumpy galaxies. There is a spiral, UDF 7036, that has the colors of a B-band drop-out, however, so it could be at $z \sim 4$ (see Sect. 6 and Table 7). Selection effects could remove the most easily recognized spirals, which are the face-on spirals, from our sample (see Sect. 5). The irregular and spiral galaxies both populate the bluer regions in Figure 5, which suggests that both types linger around in cosmological time after the spirals become obvious (see also Franceschini et al. 1998).

The ellipticals also extend in Figure 5 to the high $\mathrm{V}_{606}-\mathrm{i}_{775}$ and $\mathrm{B}_{435}-\mathrm{V}_{606}$ values of the clumpy types. The ellipticals are slighlty redder than the other types, lacking the big clump of points around $\mathrm{i}_{775}-\mathrm{Z}_{850} \sim 0$ and $\mathrm{B}_{435}-\mathrm{V}_{606} \sim 0$ that all the others have. Evidently the ellipticals have much less star formation at the epoch of observation, as is well known (e.g., Ferguson, Dickinson \& Williams 2000).

\section{Relative Numbers}

Figure 6 plots the number and fraction of galaxies of each type as a function of $i_{775}$ magnitude. The counts of galaxies with spiral or elliptical morphologies peak at about the same apparent magnitude, which is slightly brighter than the peak of the clump clusters. The counts of chain, double, and tadpole galaxies all peak at about one magnitude fainter. The fractions follow a similar trend, with spirals dominating the bright galaxies, while chains, doubles, and tadpoles dominate the faint galaxies. As is well known, the galaxies with the most unusual morphologies increase in dominance at the faintest magnitudes, beyond $\mathrm{i}_{775} \sim 26$.

The trend for chains, doubles, and tadpoles to dominate at the faintest magnitudes illustrates an important selection effect discussed in the next section. We believe on the 
basis of this trend, and on the basis of the distribution of axial ratios for spirals, that the spirals begin to disappear at $\mathrm{i}_{775} \sim 26$ because the face-on spirals become too faint to see at the surface brightness limit of the survey ( $\sim 26.0 \mathrm{mag} \operatorname{arcsec}^{-2}$ for $2 \sigma$ detections $)$. Edgeon spirals have higher surface brightnesses than face-on spirals, and are observed to fainter magnitudes. In the distribution of axial ratios (see below), there is a clear and unusual drop in the count for face-on spirals. In the same way, the chain galaxies extend to fainter magnitudes than the clump-clusters in Figure 6 because chain galaxies are probably edgeon clump clusters (Paper II). Edge-on galaxies tend to have higher surface brightnesses and fainter apparent magnitudes than face-on galaxies (see models in Paper I). Thus, the faintest galaxies at the surface-brightness limit of detection are observed to be linear structures, many of which are probably flattened objects viewed edge-on. The tadpole galaxies, for example, could be strongly interacting galaxies with a $10^{9}-10^{10} \mathrm{M}_{\odot}$ merger remnant seen as a single clump at one end and a long tidal tail seen as the diffuse lane (e.g., see Straughn et al. 2004).

\section{Axial Ratios}

Figure 7 compares the distribution of axial ratios, $W / L$, for elliptical galaxies in the UDF field with the distribution for local ellipticals in the Third Reference Catalogue of Bright Galaxies (de Vaucouleurs et al. 1991; RC3). The UDF ratios came from the ellipse fits in the published UDF catalog. The error bars equal $N^{1 / 2}$ for number $N$. For the UDF distribution, the dashed histogram is for the half of the sample with the brightest average surface brightness $\left(\mu_{I}<24.4 \mathrm{mag} \operatorname{arcsec}^{-1}\right)$. This bright half is slightly rounder on average than the faint half, as observed locally (Tremblay \& Merritt 1996). The overall distribution for UDF ellipticals resembles the distribution for local galaxies, particularly for the high surface brightness ellipticals, suggesting that elliptical galaxies relaxed to their current forms at very early times.

The distribution of axial ratios for spiral galaxies is shown in Figure 8. The spiral galaxies in the deep field background of the Tadpole Galaxy (Tran et al. 2003; Paper I) are shown on the upper left. The UDF spirals are on the bottom left. We divided the UDF spirals into the faint surface-brightness half and the bright surface-brightness half and plotted the separate distributions on the right of Figure 8. For the UDF spirals, the solid-line histograms use the ellipticities given in the UDF catalog and the dashed-line histograms use ellipticities derived individually for each galaxy from ellipse fits in IRAF. Because the spirals are irregular, we did not want to use the SExtractor ellipticities exclusively. To check the first two ellipticity measures, we also determined the axial ratios ( $W / L=1$-ellipticity) by eye, based on the length $(L)$ and width $(W)$ of the average $2-\sigma$ contour limits for each galaxy. 
All three axial ratio determinations correlated well with each other, to within 0.1 in $W / L$. In what follows we consider only the axial ratios determined by the UDF automatic ellipse fit and by our IRAF ellipse fits, which are more objective than the visual fits to contour shapes.

The distribution of axial ratios for spirals does not resemble the local distributions for spirals, which is reproduced in Figure 9 using data from the RC3. The local distribution is relatively flat from $W / L \sim 1$ down to $W / L \sim 0.1$, depending on Hubble type, indicating that the objects are round disks with intrinsic axial ratios comparable to this lower limit. The only exception is for spirals of de Vaucouleurs type 9, which are Sm irregulars and known to be relatively thick.

The UDF and Tadpole-Field spirals have a distribution of axial ratios that is peaked in the middle, which means that the catalog lacks face-on examples $(W / L \sim 1)$. The fall-off at low $W / L$ is probably from the intrinsic disk thickness, as for local galaxies, although these spiral disks are evidently $\sim 2$ times thicker than the local versions (e.g., Reshetnikov et al. 2002; Papers II, III). The fall-off at high $W / L$ could arise if the spirals are intrinsically elongated disks, i.e., not circular, as suggested for local irregular galaxies (Binggeli \& Popescu 1995; Sung et al. 1998). Alternatively, they could be circular disks that tend to be overlooked when viewed face-on. We consider these two options in detail now.

Figure 10 shows several models for the projected axial ratios of intrinsically oval, i.e., triaxial, disks. The two on the left show simple cases and the two on the right are sample fits to the UDF or Tadpole data. The model on the bottom left is for circular thick disks with a range of relative thicknesses, $Z / L=0.225$ to 0.375 , viewed in projection at random angles. The flat part extending to $W / L=1$ is a result of the circular form of the disk itself, and the rise and fall below $W / L \sim Z / L$ is for the edge-on case, when the minimum axial ratio is viewed. The top left is for an ellipsoid with an axial ratio of 1:0.6:0.2. The peak at $W / L=0.6$ is from the main disk axial ratio, when the disk is viewed face-on. The two peaks at $W / L=0.2$ and 0.33 are for edge-on versions; the lower ratio corresponds to the case where the oval disk is viewed edge-on parallel to the minor axis (showing the major axis length in projection), and the higher ratio corresponds to the case where the oval disk is viewed edge-on along the major axis (showing the minor axis length in projection). Neither of these ideal cases matches the data, but mixtures of them do. The models on the right span a range of intrinsic $W / L$ and $Z / L$ ratios, viewed in projection for orientations of the ellipsoid at random angles in 3D space.

Evidently, the distribution of axial ratios observed for the UDF is consistent with intrinsically elongated spiral disk galaxies. If the disks are actually distorted like this, then tidal forces would be a likely explanation. Such an interpretation is plausible in view of 
the importance of galaxy interactions at high redshift. However, visual examination of each UDF spiral suggests that very few of the elongated cases have companion galaxies or other direct evidence of a strong interaction. Thus we consider another explanation, as follows.

Recall that Figure 8 showed that the distribution of axial ratios for the low surface brightness spirals is slightly more skewed toward edge-on than the distribution for the high surface brightness spirals. This distortion is shown again in Figure 11, which plots the $\mathrm{i}_{775}$ magnitudes and average surface brightnesses (out to the $2 \sigma$ contour) versus the axial ratios. The solid red line is the average of the plotted points, which are from the UDF catalog. The dashed red line is the average for the $\mathrm{W} / \mathrm{L}$ values we measured based on IRAF ellipse fits (these values are not plotted). The density of points on this plot increases for intermediate $W / L$, reflecting the peak at intermediate $W / L$ in Figure 8 . The density of points shifts to even lower $W / L$ at the faintest surface brightnesses (top part of the top panel), and this shift also parallels the trend in Figure 8.

The bottom part of Figure 11 shows that the average flux gets $\sim 2$ mag brighter as the spiral galaxies become more face-on. This brightening also occurs for local spirals because the total flux increases as the projected area increases and stars become less obscured by midplane dust.

The top part of Figure 11 indicates that the average surface brightness is either constant or increases slightly ( $\sim 0.5 \mathrm{mag}$ ) for more face-on spirals, which is contrary to expectations. Locally the surface brightness decreases for face-on disks because the path length through the stars decreases (Paper I). The situation changes, however, if most of the UDF surface brightnesses for spirals are below the detection limit. This limit is $26 \mathrm{mag} \operatorname{arcsec}^{-2}$ for the faintest contour that defines our sample, so the average surface brightness of a typical galaxy inside this contour is greater, perhaps by a magnitude. The two dashed green lines in this figure represent the approximate region where we begin to lose galaxies at the surface brightness limit. Above $\sim 26 \mathrm{mag} \operatorname{arcsec}^{-2}$, the integrated surface brightness would be fainter than the lowest contour considered here $(2 \sigma)$ and the galaxy would not likely be in our survey. Thus we should ask what a distribution of surface brightnesses should look like if the average surface brightness decreases with increasing $W / L$ as for local galaxies, and as it decreases, the detection limit is passed so the fainter galaxies become lost in the noise. The solid green line shows what the average distribution of surface brightnesses should be if a face-on galaxy is at $25.9 \mathrm{mag} \operatorname{arcsec}^{-2}$ (where $W / L=1$ ) and edge-on galaxies get brighter in proportion to the path length through the disk (i.e., $\mu=25.9-2.5 \log L / W$ ). This solid line is well populated with observed galaxies at low $W / L$ so the number of points in the plot is large there, but as $W / L$ increases, the surface brightness limit comes in and the number of points centered on the line decreases. 
Another way to see this surface brightness limit is with Figure 6. There we noted that the spiral count (in the left panel) drops precipitously for galaxies fainter than $\mathrm{i}_{775} \sim 25.5$ mag, which is about where the distributions of chains, doubles, and tadpoles - all linear systems - begin to peak. This drop may be combined with a model result in Paper I to explain the factor-of-5 drop in the axial ratio distribution function between $W / L=0.5$ and 1 (Fig. 8). Paper I showed in its Figure 8 a radiative transfer model through a thick exponential disk with various levels of extinction; the average disk surface brightness of the projected disk was plotted versus the axial ratio. As $W / L$ increased from 0.5 to 1 , the average surface brightness decreased by factors of $1.92,1.78$, and 1.74 for perpendicular extinctions to the midplane at the galaxy center equal to $0.33,1.33$, and 1.77 optical depths. The limiting case with no extinction would be a factor of 2 , which is the inverse ratio of the two $W / L$ values, as this intensity ratio is mostly the ratio of path lengths along the line of sight. For these three extinctions, the surface brightness dimming in going from $W / L=0.5$ to 1 corresponds to $0.71,0.63$, and $0.61 \mathrm{mag} \operatorname{arcsec}^{-2}$. Such magnitude changes correspond, in Figure 6 , to more than half of the full drop in spiral galaxy counts (left-hand panel). This means that at the limit of the UDF survey, projection effects alone can change a galaxy that would be detected at $W / L<0.5$ to one that would be below the limit of detection at $W / L \sim 1$.

A simple model for the probability of detection of disk galaxies near the surface brightness limit of a survey is shown in Figure 12. This model assumes that the intrinsic surface brightness distribution of face-on spiral galaxies, in magnitudes $\operatorname{arcsec}^{-2}$, is Gaussian, i.e., that the distribution of intensity is log-normal. This assumption is consistent with the narrow range of surface brightnesses for modern spiral galaxies (Freeman 1970) and with the Tully \& Fisher (1977) relation, which suggests the same narrow range. In terms of the model on the top of Figure 11, the breadth of the Gaussian would give the intrinsic vertical range of points around the solid green line. For Figure 12, we integrate over this log-normal distribution to find the relative number of galaxies detected above some minimum intensity level. The absolute value of the intensity at the peak of the Gaussian is arbitrary so we take it to be $I_{f}($ peak $)=1$, and we take the Gaussian dispersion to be $\sigma$. The observed intensity of a galaxy with face-on intensity $I_{f}$ is assumed to be $I_{f} L / W$, which is approximately the case without extinction (for $W / L$ not too small). This means that if an observed intensity is $I$, then the intrinsic face-on intensity for use in the distribution function is $I W / L$. Thus we plot the detection probability:

$$
P_{\text {detect }}=\frac{\int_{\ln I_{\text {thresh }}}^{\infty} \exp \left(-0.5[\ln \{I W / L\} / \sigma]^{2}\right) d \ln I}{\int_{-\infty}^{\infty} \exp \left(-0.5[\ln \{I W / L\} / \sigma]^{2}\right) d \ln I} .
$$

The intensity threshold $I_{\text {thresh }}$ and intrinsic dispersion were chosen to match the observed fall-off in the distribution of axial ratios between $W / L=0.5$ and 1 . This gives $\ln I_{\text {thresh }}$ 
between 0.25 and 0.5 , or $I_{\text {thresh }}$ between $e^{0.25}=1.3$ and $e^{0.5}=1.6$ times the intensity at the peak of the log-normal distribution for the two cases plotted. The fit also gives $\sigma=0.2$.

The fits in Figure 12 indicate that the fall-off in spiral galaxy counts between $W / L=0.5$ and 1 corresponds to a most-probable, face-on, galaxy surface brightness that is fainter than the threshold of detection by a factor between 1.3 and 1.6. Thus a high fraction of face-on spiral galaxies are likely to be missed and high fraction of inclined spiral galaxies are likely to be seen (if they satisfy our $>10$ pixel size selection).

The fall-off in galaxy count for face-on spirals does not appear for local galaxies (Fig. 9). This is because there is a limited range in surface brightness for most spiral galaxies and the imaging surveys that made the RC3 went considerably deeper than the average galaxy surface brightness. The fact that there is also not a large increase in local counts at small $W / L$ indicates that there is not a relatively large number of thin, ultra-low surface brightness galaxies in the local Universe. The situation is apparently different in the UDF because even though galaxies tend to be intrinsically more intense than they are locally, i.e., because of their higher star formation rates, cosmological surface brightness dimming, which is proportional to $(1+z)^{4}$, knocks down their apparent surface brightnesses by large factors, corresponding to $3,4.8,6$, and 7 magnitudes $\operatorname{arcsec}^{-2}$ for $z=1,2,3$ and 4 . Thus only the brightest of the local galaxies, viewed at these redshifts, would be detected in the UDF survey, and most would be lost unless they were viewed nearly edge-on.

The distribution of axial ratios for chain and clump cluster galaxies in the UDF was shown in Paper III. It is flatter than the spiral galaxy distribution, perhaps because the clumps in clump clusters are very bright and they can still be seen in the face-on versions even if the interclump medium is close to or below the survey limit.

Figure 6 showed a large difference in the apparent magnitude distribution for spirals compared to the linear systems (chains, doubles and tadpoles) and a modest difference compared to clump clusters, which may be face-on versions of chains. The linear systems dominate at the faintest magnitudes and some of the reason for this may be the surface brightness selection effects just described, especially since the spiral sample in Figure 6 contains the full range of axial ratios. However, not all of the difference in these distributions is likely to result from surface brightness selection. Paper I showed that even the edge-on spirals in the Tadpole field, as identified by their bulges and exponential disks, tend to have brighter apparent magnitudes and slightly brighter surface brightnesses than the chains, doubles, and tadpoles. This is true also for the edge-on spirals in the UDF sample (not shown here). This difference among the purely edge-on systems could be the result of a smaller average redshift for the spirals than for the chains, doubles and tadpoles, which would be consistent with evolution over time from irregular structures to smooth spiral disks. For 
example, we noted in Paper I that the spirals in the Tadpole field tend to have slightly larger angular sizes than the doubles and tadpoles (by 50\% on average). The redshift distributions of galaxies with various morphologies will have to be studied in order to determine the relative importance of surface brightness selection and evolutionary effects.

\section{Lyman Break Galaxies}

Steidel et al. (1999 and references therein) identified high z galaxies photometrically by their redshifted Lyman break. Adelberger et al. (2004) and others applied the technique to galaxies between redshifts 1 and 3. In the UDF field, Lyman Break Galaxies (LBGs) appear as B-band drop-outs at $z \sim 4$ and V-band drop-outs at $z \sim 5$. Giavalisco et al. (2004) gave color criteria for Lyman Break galaxies, based on a sample of LBGs in the GOODS/ACS survey. Based on their criteria, we find in our UDF sample 30 B-band drop-outs (10 chains, 4 clump clusters, 8 doubles, 4 tadpoles, 1 spiral, and 3 ellipticals) and 13 V-band drop-outs (1 chain, 3 clump-clusters, 8 doubles, and 1 tadpole). These galaxies are identified by their type and UDF catalog number in Table 7. In Figure 5, many of the objects redder than $\sim 1.5$ in $B_{435}-V_{606}$ are B-band drop-outs. There are many other LBG galaxies in the UDF that are smaller than our 10 pixel major axis limit (Bouwens et al. 2004). Bunker et al. (2004) and Yan \& Windhorst (2004) reported over 100 i-band drop-out $(z \sim 6)$ galaxies in the UDF; one of our V-band drop-out chain galaxies was also reported at redshift $\mathrm{z}=5.4$ by Rhoads et al. (2004).

Most of the drop-outs in Table 7 are linear galaxies (doubles, chains, tadpoles) and relatively few are disks (spiral). This is consistent with the common perception that disks form late, but it may also contain a selection bias like that discussed in the previous section if most face-on disks are fainter than the survey limit.

Madau et al. (1998) determined star formation rates (SFRs) from models of star clusters with Salpeter initial mass functions based on observed UV fluxes. Using their conversions, and assuming that the rest wavelength of $1500 \AA$ is shifted approximately to $\mathrm{i}_{775}$-band for the $z \sim 4$ galaxies and to $\mathrm{Z}_{850}$ band for $z=5$, we calculated the SFRs (in $\mathrm{M}_{\odot} /$ year) of our LBGs. The results are listed in Table 7 . The star formation rates for the V-band drop-outs in this sample tend to be larger than for the B-band drop-outs. In comparison, Immeli et al. (2004) predicted a SFR of about $20 \mathrm{M}_{\odot} \mathrm{yr}^{-1}$ from their chain galaxy models, which assumed galaxy sizes a factor of five larger than those of our LBGs. Somerville, Primack, \& Faber (2001) got rates of $\sim 1-30 \mathrm{M}_{\odot} \mathrm{yr}^{-1}$ for the largest galaxies considered in their Lyman Break models. 


\section{Summary}

We classified and measured 884 galaxies larger than 10 pixels in the Hubble UDF. Six prominent types were used: chain, clump-cluster, double, tadpole, spiral, and elliptical. Their colors, magnitudes, and surface brightnesses are similar, reflecting their likely formation over a large range of times. The distribution of axial ratios for elliptical galaxies is similar to that in the local field, suggesting that the elliptical shapes were quickly established and remained unchanged over time. The distribution of axial ratios for spiral galaxies

was distinctly different than for local galaxies with a deficit of face-on spirals in the deep field. Models illustrate how this could be the result of an intrinsically oval shape, as might be expected for highly perturbed galaxies. However, other models suggest that most of the face-on spirals could be lost below the surface brightness limit of the survey, while the more highly inclined spirals could still be seen. A significant lack of spiral galaxies with low axial ratios implies an intrinsic disk thickness of 0.2 to 0.3 times the disk length. This implies the spiral disks are thicker than local spirals by a factor of $\sim 2$ to 3 .

Twenty-six barred spiral galaxies were found, a fraction of the total spirals equal to about $10 \%$. This is a factor of 2 less than other deep field studies but not inconsistent with them considering projection effects and surface brightness dimming. In addition, bars become harder to distinguish at high redshifts because the spiral galaxies generally get more irregular.

We thank the Vassar College URSI program and the Keck Northeast Astronomy Consortium exchange program for undergraduate research support for M.S. and D.S.R.; B.G.E. is supported by the National Science Foundation through grant AST-0205097. 
Table 1: Chain Galaxies

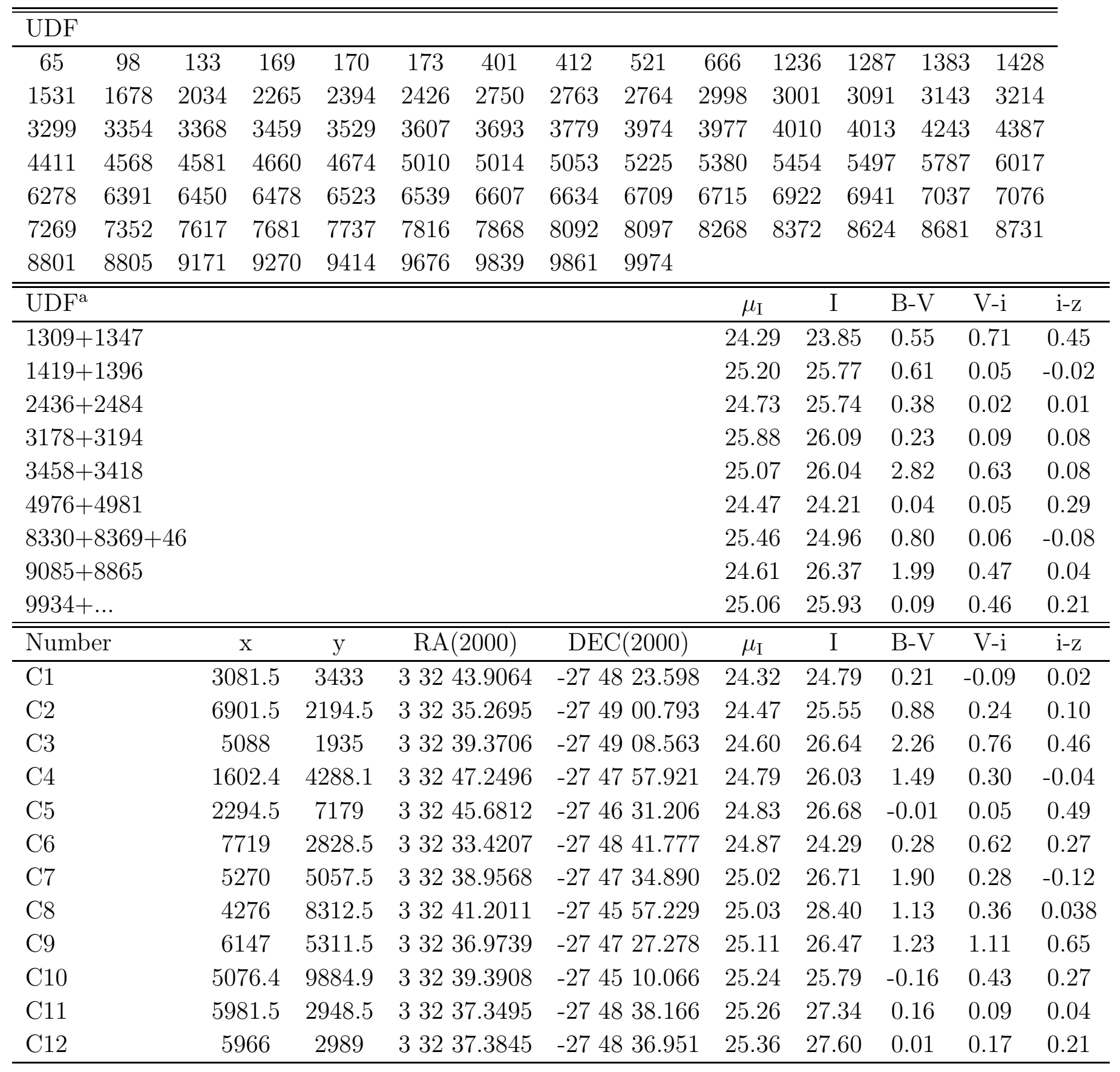

${ }^{a}$ UDF 9934 also includes UDF 9912 and UDF 9949. 
Table 2: Clump Cluster Galaxies

\begin{tabular}{|c|c|c|c|c|c|c|c|c|c|c|}
\hline \multicolumn{11}{|l|}{$\mathrm{UDF}$} \\
\hline 40 & 84 & 97 & 126 & 168 & 187 & 533 & 551 & 566 & 769 & 791 \\
\hline 918 & 941 & 1041 & 1112 & 1266 & 1269 & 1316 & 1362 & 1375 & 1589 & 1666 \\
\hline 1859 & 2012 & 2174 & 2240 & 2291 & 2340 & 2350 & 2350 & 2463 & 2499 & 2538 \\
\hline 3037 & 3154 & 3243 & 3270 & 3460 & 3483 & 3688 & 3689 & 3745 & 3752 & 3778 \\
\hline 3881 & 4006 & 4084 & 4093 & 4094 & 4253 & 4262 & 4370 & 4616 & 4807 & 4860 \\
\hline 5136 & 5190 & 5201 & 5216 & 5491 & 5501 & 5548 & 5579 & 5620 & 5634 & 5683 \\
\hline 5837 & 5860 & 5878 & 5896 & 5946 & 6056 & 6133 & 6187 & 6394 & 6396 & 6438 \\
\hline 6645 & 6710 & 6785 & 6821 & 6837 & 6854 & 6939 & 6943 & 7185 & 7227 & 7230 \\
\hline 7559 & 7647 & 7678 & 7725 & 7756 & 7786 & 7905 & 7995 & 8022 & 8042 & 8125 \\
\hline 8314 & 8461 & 8551 & 8682 & 8710 & 8749 & 8765 & 8802 & 8859 & 8880 & 9000 \\
\hline 9299 & 9347 & 9356 & 9396 & 9409 & 9474 & 9837 & 9853 & & & \\
\hline \multicolumn{6}{|c|}{$\mathrm{UDF}^{\mathrm{a}}$} & $\mu_{\mathrm{I}}$ & $\mathrm{I}$ & $\overline{B-V}$ & $\mathrm{~V}-\mathrm{i}$ & $\mathrm{i}-\mathrm{Z}$ \\
\hline \multicolumn{5}{|c|}{$82+99+103$} & & 25.07 & 23.59 & 0.43 & 0.25 & 0.30 \\
\hline \multicolumn{5}{|c|}{$3034+3129$} & & 24.6 & 24.03 & 0.82 & 0.18 & 0.08 \\
\hline \multicolumn{5}{|c|}{$3465+3597$} & & 24.32 & 23.48 & 0.11 & 0.04 & 0.02 \\
\hline \multicolumn{5}{|c|}{$4245+\ldots$} & & 26.16 & 25.08 & 0.08 & 0.25 & 0.32 \\
\hline \multicolumn{5}{|c|}{$4258+4458$} & & 25.12 & 25.56 & 0.60 & 0.27 & 0.10 \\
\hline \multicolumn{5}{|c|}{$4765+4795$} & & 24.81 & 25.56 & 2.03 & 0.38 & -0.01 \\
\hline \multicolumn{5}{|c|}{$4801+5271$} & & 25.17 & 23.87 & 0.22 & 0.37 & 0.56 \\
\hline \multicolumn{5}{|c|}{$6235+6153$} & & 25.14 & 25.59 & 0.01 & 0.17 & 0.33 \\
\hline \multicolumn{5}{|c|}{$6462+6886$} & & 25.51 & 23.48 & 0.18 & 0.32 & 0.27 \\
\hline \multicolumn{5}{|c|}{$6713+6738$} & & 25.24 & 26.35 & 3.86 & 0.62 & -0.08 \\
\hline & & \multicolumn{3}{|c|}{$7008+7045$} & & 25.35 & 26.16 & 1.31 & 0.32 & 0.08 \\
\hline & & \multicolumn{3}{|c|}{$7081+\ldots$} & & 25.86 & 24.52 & 0.06 & 0.105 & 0.34 \\
\hline & & \multicolumn{3}{|c|}{$9169+9332$} & & 24.88 & 24.48 & 0.16 & 0.18 & 0.30 \\
\hline & & \multicolumn{3}{|c|}{$9166+9102$} & & 25.13 & 24.35 & 0.32 & 0.63 & 0.62 \\
\hline
\end{tabular}

\begin{tabular}{cccccccccc}
\hline \hline Number & $\mathrm{x}$ & $\mathrm{y}$ & $\mathrm{RA}(2000)$ & $\mathrm{DEC}(2000)$ & $\mu_{\mathrm{I}}$ & $\mathrm{I}$ & $\mathrm{B}-\mathrm{V}$ & $\mathrm{V}-\mathrm{i}$ & $\mathrm{i}-\mathrm{z}$ \\
\hline $\mathrm{CC} 1$ & 4446 & 1030 & 33240.8232 & -274935.706 & 24.32 & 24.11 & 1.20 & 0.24 & 0.05 \\
$\mathrm{CC} 2$ & 3809.2 & 1717.2 & 33242.2627 & -274915.082 & 25.16 & 24.52 & 0.88 & 0.12 & 0.21 \\
$\mathrm{CC} 3$ & 6154.5 & 2458 & 33236.9586 & -274852.883 & 25.14 & 25.90 & 0.056 & 0.45 & 0.09 \\
$\mathrm{CC} 4$ & 688 & 5339 & 33249.3154 & -274726.377 & 23.86 & 22.00 & 0.44 & 0.16 & -0.07 \\
$\mathrm{CC} 5$ & 769.3 & 4832.3 & 33249.1323 & -274741.579 & 25.36 & 25.23 & 0.02 & 0.26 & 0.08 \\
$\mathrm{CC} 6$ & 1117 & 5932.5 & 33248.3447 & -274708.580 & 25.13 & 26.14 & 0.81 & 0.04 & -0.21 \\
$\mathrm{CC} 7$ & 1168.7 & 6154.3 & 33248.2275 & -274701.927 & 24.82 & 26.47 & 0.49 & 0.04 & -0.18 \\
CC8 & 8296.5 & 6395 & 33232.1142 & -274654.784 & 24.15 & 25.13 & 0.99 & 0.24 & 0.03 \\
CC9 & 9018 & 6086 & 33230.4832 & -274704.055 & 24.69 & 26.20 & 0.17 & 0.04 & 0.02 \\
CC10 & 8824 & 6572 & 33230.9217 & -274649.475 & 24.62 & 24.18 & 0.75 & 0.87 & 0.72
\end{tabular}


Table 3: Double Galaxies

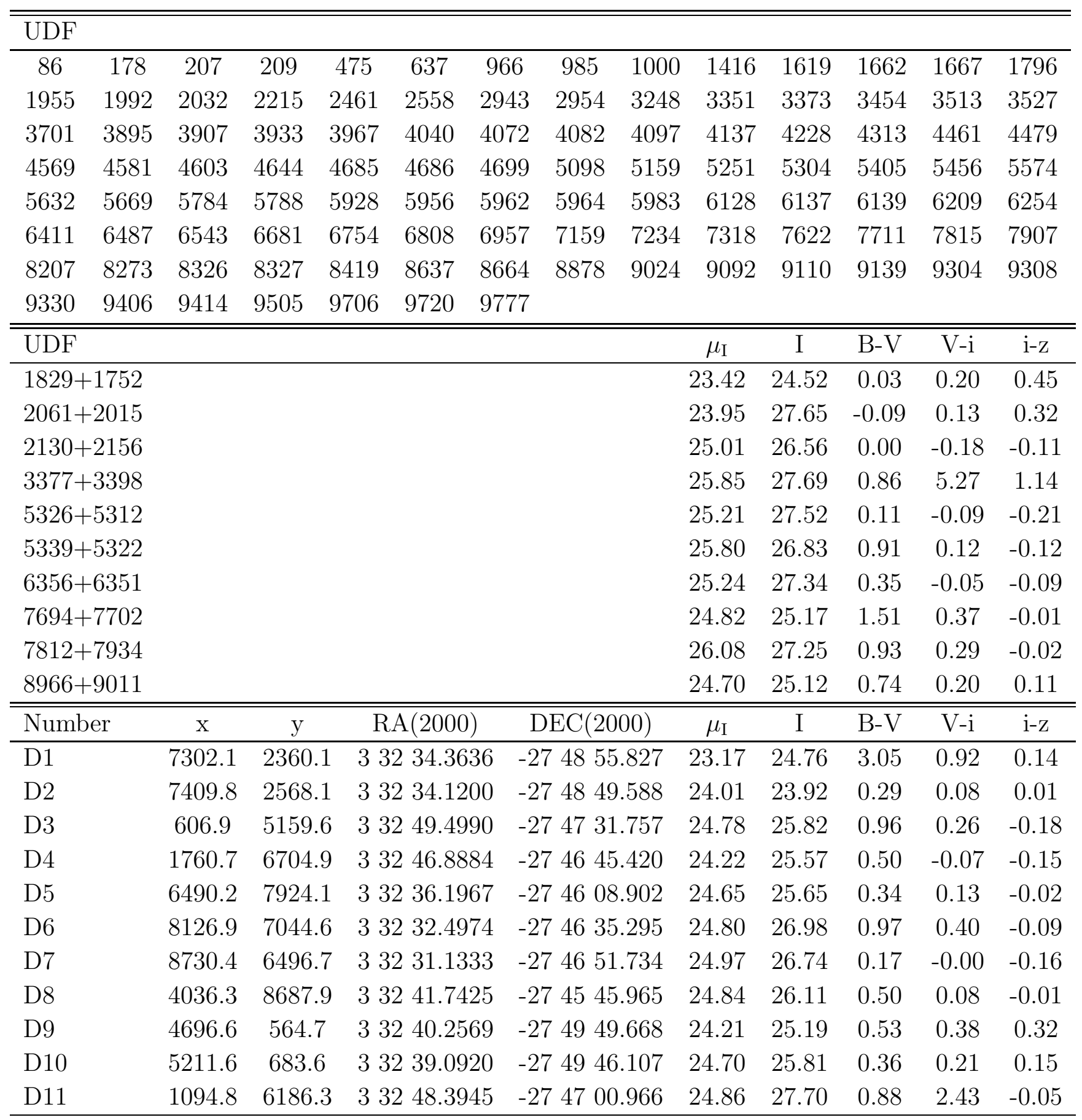


Table 4: Tadpole Galaxies

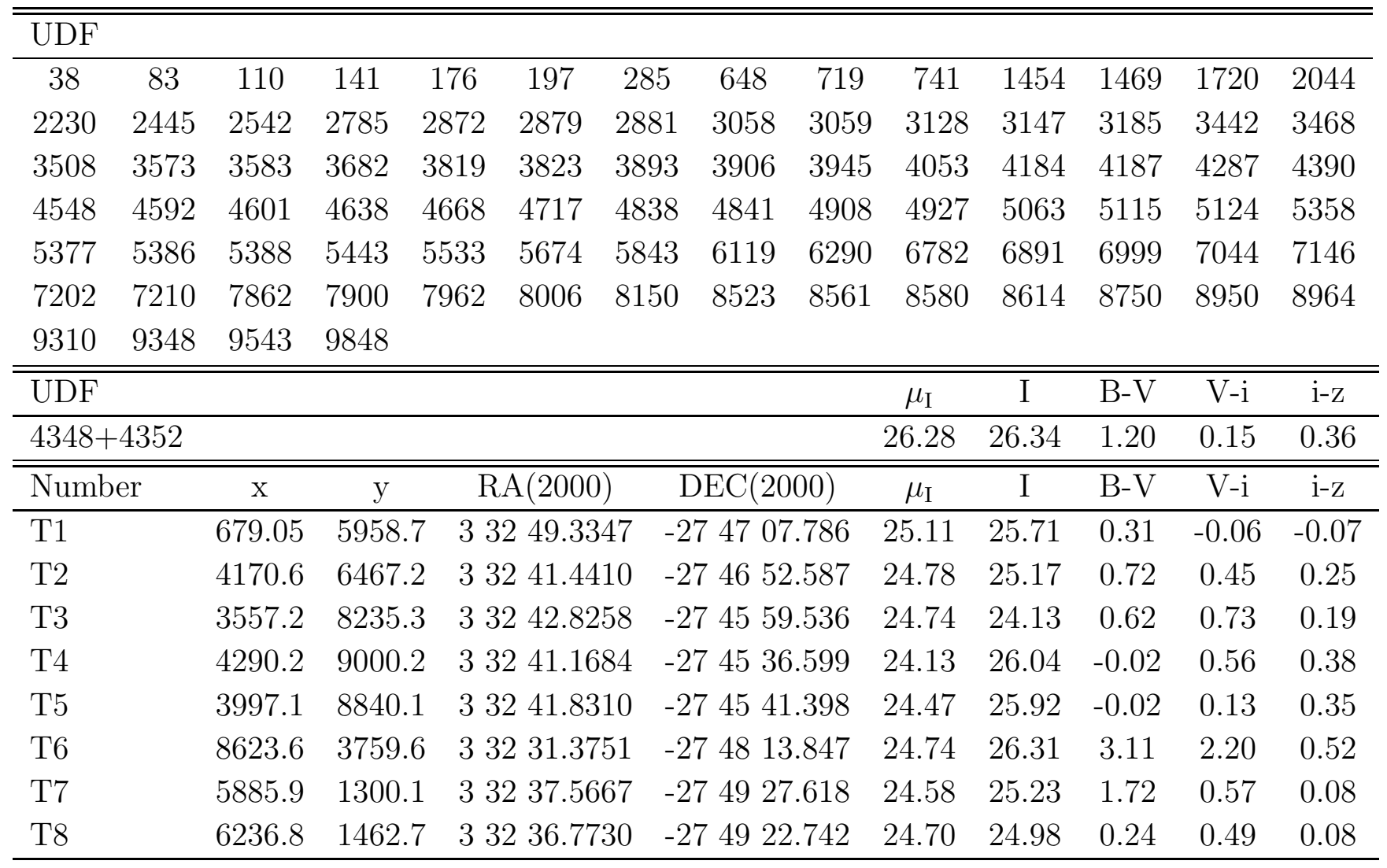


Table 5: Spiral Galaxies ${ }^{\mathrm{a}}$

\begin{tabular}{|c|c|c|c|c|c|c|c|c|c|c|c|c|}
\hline UDF & & & & & & & & & & & & \\
\hline 1 & 2 & 13 & 14 & 15 & 17 & 20 & 37 & 43 & 50 & 55 & 95 & 131 \\
\hline 192 & 213 & 235 & 237 & 295 & 328 & 355 & 423 & 424 & 428 & 446 & 501 & 503 \\
\hline 542 & 656 & 662 & 679 & 697 & 735 & 833 & 898 & 916 & 968 & 1006 & 1049 & 1052 \\
\hline 1270 & 1403 & 1421 & 1426 & 1478 & 1551 & 1571 & 1592 & 1612 & 1626 & 1668 & 1732 & 1739 \\
\hline 1809 & 1830 & 1889 & 1898 & 1904 & 1905 & 1922 & $1971 *$ & 2017 & 2104 & 2170 & 2189 & 2306 \\
\hline 2333 & 2358 & 2462 & 2525 & 2607 & 2652 & 2753 & 2799 & 2813 & 2993 & 3006 & 3013 & 3068 \\
\hline 3097 & 3101 & 3123 & 3180 & $3203^{*}$ & 3247 & 3257 & 3268 & 3315 & 3319 & $3349 *$ & 3372 & 3422 \\
\hline 3472 & $3492^{*}$ & 3610 & $3613^{*}$ & 3680 & 3785 & $3822 *$ & 3823 & 3840 & 3871 & 4052 & 4058 & 4192 \\
\hline 4315 & 4321 & 4360 & 4394 & 4407 & 4410 & 4438 & 4478 & 4491 & 4591 & 4661 & 4662 & 4767 \\
\hline 4835 & 4878 & 4907 & 4929 & 4950 & 5022 & $5177^{*}$ & 5268 & 5276 & 5286 & $5365^{*}$ & 5408 & 5411 \\
\hline 5435 & 5473 & 5505 & 5540 & 5569 & $5606^{*}$ & 5614 & 5615 & 5658 & 5670 & 5694 & 5697 & 5753 \\
\hline 5812 & 5864 & $5898 \mathrm{~A}^{\mathrm{b}}$ & $5898 \mathrm{~B}^{\mathrm{b}}$ & 5922 & 5989 & 5995 & 5999 & 6008 & 6038 & $6051 *$ & 6060 & 6079 \\
\hline 6107 & 6143 & 6188 & 6203 & 6206 & 6639 & 6674 & 6680 & 6810 & 6834 & $6862^{*}$ & 6911 & 6933* \\
\hline 6997* & 7022 & 7036 & 7067 & 7071 & 7112 & $7203^{*}$ & 7406 & $7452^{*}$ & 7495 & 7556 & 7664 & $7688^{*}$ \\
\hline 7743 & 7775 & 7847 & 7974 & 8015 & $8026^{*}$ & 8040 & $8049^{*}$ & 8051 & 8156 & 8181 & 8255 & 8257 \\
\hline 8261 & 8275 & 8351 & 8409 & 8454 & 8576 & 8585 & 8603 & 8629 & 8693 & 8757 & 8782 & 8918 \\
\hline 9074 & 9125 & 9183 & 9204 & 9253 & 9341* & 9425 & $9444^{*}$ & 9455 & 9599 & 9609 & 9649 & 9724 \\
\hline 9807 & 9834 & 9895 & & & & & & & & & & \\
\hline
\end{tabular}

\begin{tabular}{|c|c|c|c|c|c|c|c|c|c|}
\hline Number & $\mathrm{x}$ & $\mathrm{y}$ & $\mathrm{RA}(2000)$ & $\operatorname{DEC}(2000)$ & $\mu_{\mathrm{I}}$ & I & $\mathrm{B}-\mathrm{V}$ & $\mathrm{V}-\mathrm{i}$ & $\mathrm{i}-\mathrm{Z}$ \\
\hline s1* & 5102.6 & 538.9 & 33239.3387 & -274950.446 & 24.54 & 23.59 & 0.78 & 0.61 & 0.19 \\
\hline s2 & 6144.9 & 1292.1 & 33236.9810 & -274927.860 & 24.50 & 24.92 & 0.18 & 0.59 & 0.17 \\
\hline s3 & 6211.3 & 1497.7 & 33236.8307 & -27 4921.692 & 25.29 & 25.56 & 0.21 & 0.71 & 0.31 \\
\hline s4 & 2806.1 & 2886.6 & 33244.5297 & -274839.986 & 24.17 & 24.43 & 0.88 & 0.28 & 0.21 \\
\hline s5 & 3916.9 & 2413.6 & 33242.0184 & -274854.192 & 25.35 & 25.63 & 1.25 & 0.92 & 0.16 \\
\hline s6 & 5150.1 & 2557.2 & 33239.2298 & -274849.898 & 24.73 & 25.63 & 0.67 & 0.09 & 0.04 \\
\hline s7 & 7100.9 & 2372.6 & 33234.8186 & -274855.451 & 24.55 & 24.48 & 0.76 & 0.20 & 0.10 \\
\hline s8 & 7845.6 & 3062.1 & 33233.1344 & -274834.769 & 24.82 & 23.85 & 0.58 & 0.65 & 0.14 \\
\hline s9 & 1982.1 & 3846.9 & 33246.3917 & -274811.164 & 25.58 & 25.72 & 0.20 & 0.01 & -0.09 \\
\hline s10 & 1958.1 & 3902.1 & 33246.4459 & -274809.507 & 24.96 & 25.09 & 1.14 & 0.60 & 0.09 \\
\hline s11 & 1640.9 & 4158.1 & 33247.1627 & -274801.822 & 25.03 & 25.36 & 1.05 & 0.26 & -0.01 \\
\hline s12 & 8265.1 & 3406.9 & 33232.1858 & -274824.427 & 25.19 & 23.60 & 0.91 & 0.23 & 0.18 \\
\hline s13* & 9257.4 & 4315.4 & 33229.9420 & -274757.173 & 23.96 & 22.68 & 1.84 & 1.21 & 0.48 \\
\hline s14 & 9193.8 & 4319.4 & 33230.0858 & -274757.053 & 24.77 & 25.53 & 0.29 & 0.11 & 0.04 \\
\hline s15 & 1120.9 & 4920.6 & 33248.3373 & -274738.937 & 24.95 & 24.55 & 0.88 & 0.17 & 0.04 \\
\hline s16 & 846.4 & 5071.1 & 33248.9577 & -274734.417 & 24.97 & 25.34 & 0.92 & 0.22 & 0.04 \\
\hline $\mathrm{s} 17^{*}$ & 9372.6 & 5878.6 & 33229.6816 & -274710.277 & 24.32 & 23.57 & 1.22 & 0.99 & 0.31 \\
\hline s18 & 9350.6 & 6065.6 & 33229.7313 & -274704.667 & 25.01 & 24.94 & 1.23 & 0.42 & 0.21 \\
\hline s19 & 5224.5 & 7207.5 & 33239.0581 & -274630.390 & 23.57 & & & 0.31 & 0.17 \\
\hline
\end{tabular}


Table 6: Elliptical Galaxies

\begin{tabular}{|c|c|c|c|c|c|c|c|c|c|c|c|c|c|}
\hline \multicolumn{14}{|l|}{$\mathrm{UDF}$} \\
\hline 8 & 25 & 36 & 68 & 97 & 100 & 153 & 176 & 191 & 206 & 221 & 287 & 310 & 582 \\
\hline 600 & 631 & 703 & 731 & 865 & 900 & 901 & 1088 & 1344 & 1358 & 1453 & 1481 & 1564 & 1607 \\
\hline 1727 & 1960 & 2016 & 2063 & 2107 & 2162 & 2201 & 2245 & 2248 & 2293 & 2322 & 2329 & 2387 & 2414 \\
\hline 2518 & 2927 & 2974 & 3048 & 3088 & 3136 & 3174 & 3288 & 3332 & 3333 & 3475 & 3591 & 3677 & 3869 \\
\hline 4096 & 4142 & 4320 & 4350 & 4389 & 4396 & 4445 & 4481 & 4527 & 4551 & 4587 & 4805 & 4913 & 5187 \\
\hline 5263 & 5959 & 6018 & 6022 & 6027 & 6047 & 6288 & 6747 & 6853 & 6853 & 7121 & 7398 & 7780 & 8069 \\
\hline 8138 & 8316 & 8680 & 9090 & 9264 & 9532 & 9765 & 9778 & 9847 & 9962 & & & & \\
\hline \multicolumn{2}{|c|}{ Number } & $\mathrm{x}$ & $\mathrm{y}$ & \multicolumn{2}{|c|}{ RA(2000) } & \multicolumn{2}{|c|}{ DEC(2000) } & $\mu_{\mathrm{I}}$ & I & $\mathrm{B}-\mathrm{V}$ & $\mathrm{V}-\mathrm{i}$ & \multicolumn{2}{|l|}{$\mathrm{i}-\mathrm{z}$} \\
\hline E1 & \multicolumn{2}{|c|}{4154.8} & 1231.1 & \multicolumn{2}{|c|}{33241.4815} & \multicolumn{2}{|c|}{-274928.72} & 24.92 & 26.05 & 0.36 & 0.62 & \multicolumn{2}{|l|}{0.20} \\
\hline $\mathrm{E} 2$ & \multicolumn{2}{|c|}{6004.4} & 1086.9 & \multicolumn{2}{|c|}{33237.2988} & \multicolumn{2}{|c|}{-274934.02} & 24.75 & 25.42 & 0.40 & 0.62 & \multicolumn{2}{|l|}{-0.19} \\
\hline E3 & \multicolumn{2}{|c|}{9026.7} & 4118.9 & \multicolumn{2}{|c|}{33230.4637} & \multicolumn{2}{|c|}{-274803.07} & 24.24 & 23.52 & 1.15 & 0.49 & \multicolumn{2}{|l|}{0.15} \\
\hline E4 & \multicolumn{2}{|c|}{3674.5} & 8546.7 & \multicolumn{2}{|c|}{33242.5604} & \multicolumn{2}{|c|}{-274550.20} & 23.56 & 22.36 & 0.28 & 1.04 & \multicolumn{2}{|l|}{0.18} \\
\hline E5 & \multicolumn{2}{|c|}{5741.9} & 9595.4 & \multicolumn{2}{|c|}{33237.8870} & \multicolumn{2}{|c|}{-274518.76} & 23.80 & 23.79 & 0.35 & 0.86 & \multicolumn{2}{|l|}{0.29} \\
\hline E6 & \multicolumn{2}{|c|}{4826.6} & 9687.6 & \multicolumn{2}{|c|}{33239.9555} & \multicolumn{2}{|c|}{-274515.98} & 25.13 & 25.75 & 0.60 & 0.30 & 0.14 & \\
\hline
\end{tabular}


Table 7: Lyman Break Drop-out Galaxies ${ }^{\mathrm{a}}$

\begin{tabular}{lclclc}
\hline \hline B-band drop-outs & & & & & \\
\hline UDF & SFR $^{\mathrm{b}}$ & UDF & SFR $^{\mathrm{b}}$ & UDF $^{\mathrm{b}}$ & SFR $^{\mathrm{b}}$ \\
\hline $65(\mathrm{C})$ & 7.9 & $141(\mathrm{~T})$ & 9.5 & $401(\mathrm{C})$ & 21 \\
$631(\mathrm{E})$ & 8.4 & $985(\mathrm{D})$ & 5.7 & $2394(\mathrm{C})$ & 3.7 \\
$3001(\mathrm{C})$ & 2.0 & $3458+3418(\mathrm{C})$ & 13 & $3778(\mathrm{CC})$ & 19 \\
$4313(\mathrm{D})$ & 4.1 & $4548(\mathrm{~T})$ & 7.7 & $4551(\mathrm{E})$ & 16 \\
$4685(\mathrm{D})$ & 14 & $4765+4795(\mathrm{CC})$ & 21 & $5548(\mathrm{CC})$ & 12 \\
$6137(\mathrm{D})$ & 2.3 & $6209(\mathrm{D})$ & 1.5 & $6450(\mathrm{C})$ & 3.8 \\
$6543(\mathrm{D})$ & 15 & $6709(\mathrm{C})$ & 1.1 & $6808(\mathrm{D})$ & 4.8 \\
$7036(\mathrm{~S})$ & 77 & $7044(\mathrm{~T})$ & 3.1 & $8092(\mathrm{C})$ & 16 \\
$8419(\mathrm{D})$ & 6.6 & $9085+8865(\mathrm{C})$ & 9.7 & $9310(\mathrm{~T})$ & 1.8 \\
C7(C) & 7.1 & E19.47(E) & 15 & $\mathrm{CC} 11(\mathrm{CC})$ & 61 \\
\hline V-band drop-outs & & & & & \\
\hline 1796(D) & 6.2 & $2350(\mathrm{CC})$ & 42 & $2881(\mathrm{~T})$ & 58 \\
$3377+3398(\mathrm{D})$ & 30 & $5225(\mathrm{C})$ & 48 & $5928(\mathrm{D})$ & 6.5 \\
$6139(\mathrm{D})$ & 63 & $6681(\mathrm{D})$ & 24 & $7328(\mathrm{CC})$ & 465 \\
8326(D) & 18 & $8664(\mathrm{D})$ & 23 & $8682(\mathrm{CC})$ & 50 \\
D11(D) & 9.9 & & & &
\end{tabular}

\footnotetext{
${ }^{a}$ initials for each morphological type are given: $\mathrm{C}=$ chain, $\mathrm{CC}=$ clump cluster, $\mathrm{D}=$ double, $\mathrm{T}=$ tadpole, $\mathrm{S}=$ spiral, $\mathrm{E}=$ elliptical

${ }^{b}$ Star formation rates in $\mathrm{M}_{\odot} \mathrm{yr}^{-1}$
} 


\section{REFERENCES}

Abraham, R., Tanvir, N., Santiago, B., Ellis, R., Glazebrook, K., \& van den Bergh, S. 1996a, MNRAS, 279, L47

Abraham, R., van den Bergh, S., Glazebrook, K., Ellis, R., Santiago, B., Surma, P., \& Griffiths, R. 1996b, ApJS, 107, 1

Adelberger, K., Steidel, C., Shapley, A., Hunt, M., Erb, D., Reddy, N., \& Pettini, M. 2004, ApJ, 607, 226

Athanassoula, E., \& Bosma, A. 2003, ApSpSci, 284, 491

Beckwith, S.V.W. et al., 2005, in preparation

Binggeli, B., \& Popescu, C. C. 1995, A\&A, 298, 63

Bouwens, R.J., Illingworth, G.D., Blakeslee, J.P., Broadhurst, T.J., \& Franx, M. 2004 ApJ, 611, L1

Brinchmann, J., et al. 1998, ApJ, 499, 112

Bruzual, G. \& Charlot, S. 2003, MNRAS, 344, 1000

Bunker, A., Stanway, E., Ellis, R., \& McMahon, R.2004, MNRAS, 355, 374

Conselice, C. 2004, astro-ph/0405102

Conselice, C., Grogin, N.A., Jogee, S., Lucas, R.A., Dahlen, T., de Mello, D., Gardner, J.P., Mobasher, B., Ravindranath, S. 2004, ApJ, 600, L139

Cowie, L., Hu, E., \& Songaila, A. 1995, AJ, 110, 1576

Dalcanton, J.J., \& Schectman, S.A. 1996, ApJ, 465, L9

Dickinson, M. 2000, Philosophical Transactions of the Royal Society of London, Series A, Vol. 358, p.2001

Elmegreen, B.G., Elmegreen, D.M., \& Hirst, A.C. 2004b, ApJ, 612, 191 (Paper V)

Elmegreen, B.G., \& Elmegreen, D.M. 2005, ApJ, 627, 632 (Paper III)

Elmegreen, D.M., Elmegreen, B.G., \& Sheets, C.M. 2004, ApJ, 603, 74 (Paper I)

Elmegreen, D.M., Elmegreen, B.G., \& Hirst, A.C. 2004a, ApJ, 604, L21 (Paper II) 
Elmegreen, D.M., Elmegreen, B.G., \& Ferguson, T.E., 2005, ApJL, 623, L71 (Paper IV)

Ferguson, H. C., Dickinson, M., \& Williams, R. 2000, ARA\&A, 38, 667

Franceschini, A., Silva, L., Fasano, G., Granato, G.L., Bressan, A., Arnouts, S., Danese, L. 1998, ApJ, 506, 600

Freeman, K.C. 1970, ApJ, 160, 811

Giavalisco, M. et al. 2004, ApJ, 600, 103

Immeli, A., Samland, M., Westera, P., \& Gerhard, O. 2004, ApJ, 611, 201

Jogee, S. et al. 2004, ApJL, 615, 105

Madau, P., Pozzetti, L., \& Dickinson M., 1998, ApJ, 106, 116

O’Neil, K., Bothun, G.D., \& Impey, C.D. 2000, ApJS, 128, 99

Reshetnikov, V., Battaner, E., Combes, F., \& Jiménez-Vicente, J. 2002, A\&A, 382, 513

Reshetnikov, V., Dettmar, R.-J., \& Combes, F. 2003, A\&A, 399, 879

Rhoads, J. et al. 2005, ApJ, 621, 582

Sheth, K., Regan, M.W., Scoville, N.Z., \& Strubbe, L.E. 2003, ApJ, 592, 13

Somerville, R.S., Primack, J.R., Faber, S.M. 2001, MNRAS, 320, 504

Steidel, C., Adelberger, K., Giavalisco, M., Dickinson, M., \& Pettini, M. 1999, ApJ, 519, 1

Straughn, A., Ryan, E., Cohen, S., Hathi, N., Windhorst, R., \& Pasquali, A. 2004, BAAS, 205, 9417

Sung, E.-C., Han, C., Ryden, B. S., Patterson, R. J., Chun, M.-S., Kim, H.-I., Lee, W.-B., \& Kim, D.-J. 1998, ApJ, 505, 199

Thompson, R. et al. 2005, astroph/053504

Tran, H. et al. 2003, ApJ, 585, 750

Tremblay, B. \& Merritt, D. 1996, AJ, 111, 2243

Tully, R. B., \& Fisher, J. R. 1977, A\&A, 54, 661

van den Bergh, S. 2002, PASP, 114, 797 
van den Bergh, S., Abraham, R.G., Ellis, R.S., Tanvir, N.R., Santiago, B.X., \& Glazebrook, K.G. 1996, AJ 112, 359

van den Bergh, S., Cohen, J.G., Hogg, D. W., \& Blandford, R. 2000, AJ, 120, 2190

van den Bergh, S., Abraham, R.G., Whyte, L.F., Merrifield, M.R., Eskridge, P.B., Frogel, J.A., \& Pogge, R. 2002, AJ, 123, 2913

de Vaucouleurs, G., de Vaucouleurs, A., Corwin, H., Buta, R., Paturel, G., \& Fouque, P. 1991, Third Reference Catalogue of Galaxies, New York: Springer-Verlag

Volonteri, M., Saracco, P., \& Chincarini, G. 2000, A\&AS, 145, 111

Williams, R., et al. 1996, AJ, 112, 1335

Yan, H., \& Windhorst, R.A. 2004, ApJL, 612, 93 

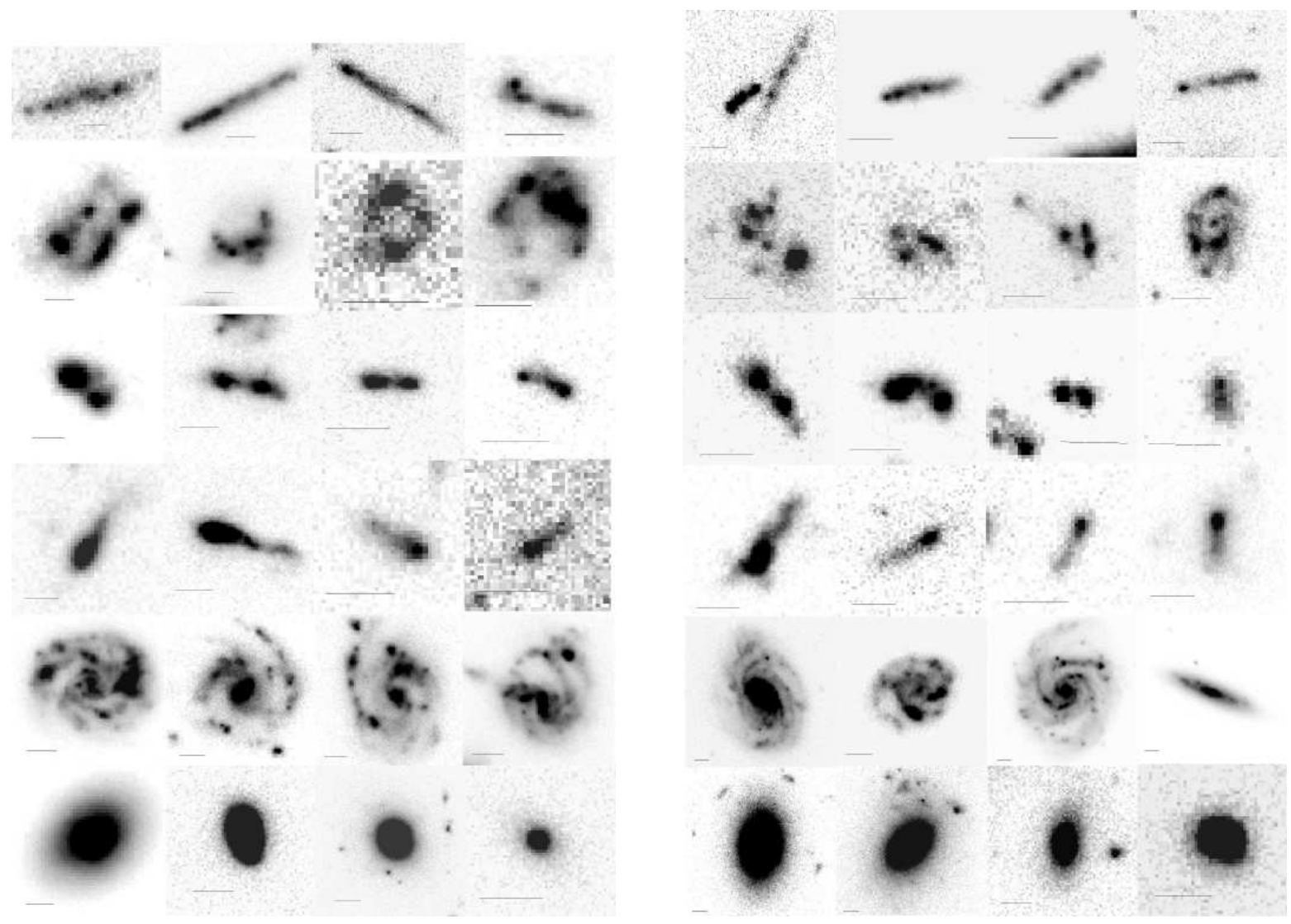

Fig. 1. - A selection of 8 typical galaxies for each morphological type: 4 in Fig a and 4 in Fig b. From top to bottom: chain, clump-cluster, double, tadpole, spiral, and elliptical. Images are at $i_{775}$-band, with a line representing 0.5 arcsec. UDF or our own identification numbers from left to right in Fig a are as follows: chains: 6478, 7269, 6922, 3214; clump clusters: CC12, 1375, 2291, 5190; doubles: 637, 4072, 5098, 5251; tadpoles: 3058, 8614, 5358, 6891; spirals: 3372, 3180, 4438, 8275; ellipticals: 2107, 4389, 2322, 4913. In Fig b, the identifications are: chains: 169 and 170 (2 separate galaxies), 1428, 401, 3458+3418; clump clusters: 6486, 4807, 7230, 9159; doubles: 2461, 2558, 4097, 3967; tadpoles: 9543, 5115, 3147, 9348; spirals: 2607, 5805, 7556, 5670; ellipticals: 8, 4527, 4320, 5959. Fig. b has an example of an edge-on spiral. 

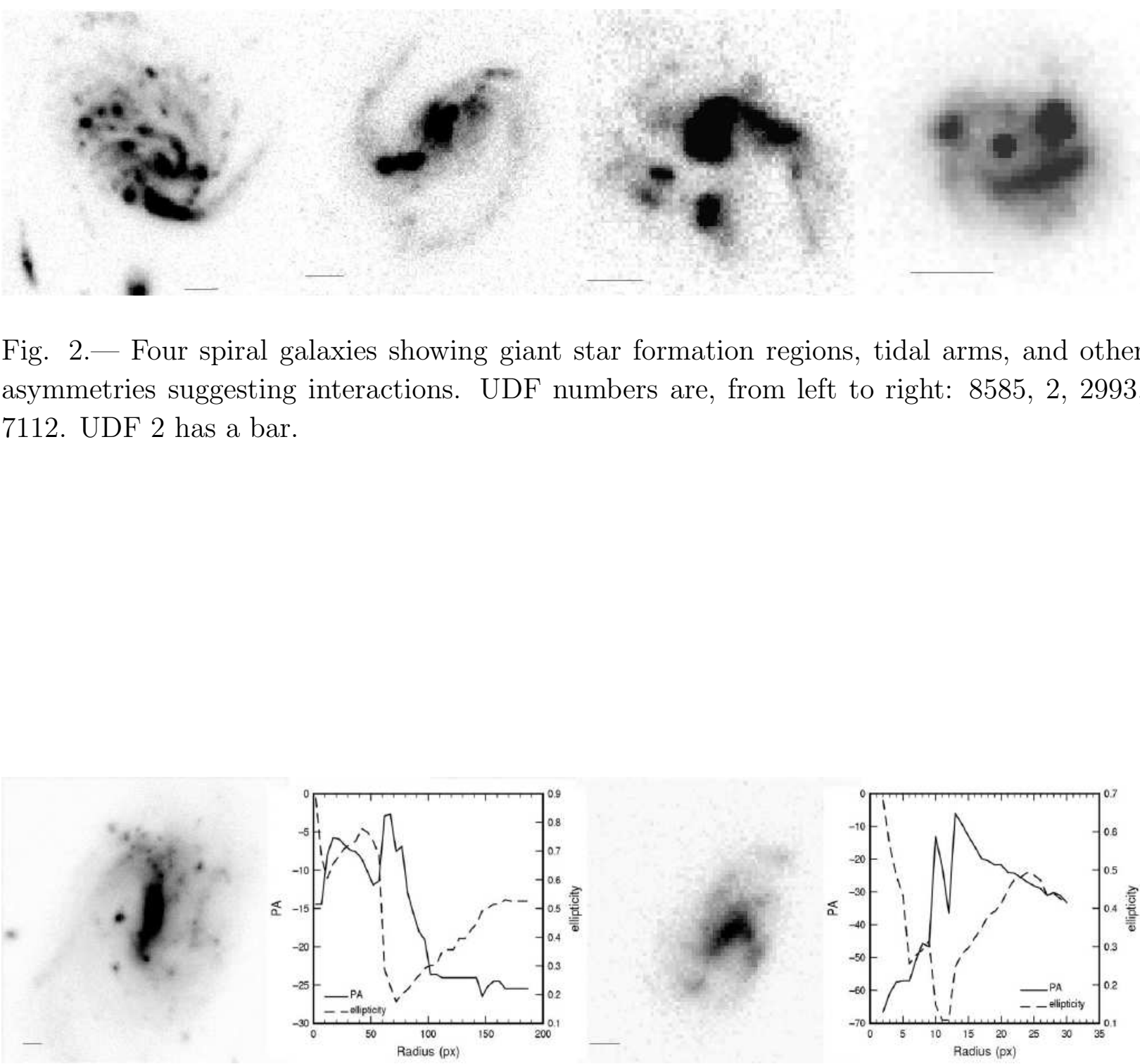

Fig. 2.- Four spiral galaxies showing giant star formation regions, tidal arms, and other asymmetries suggesting interactions. UDF numbers are, from left to right: 8585, 2, 2993, 7112. UDF 2 has a bar.

Fig. 3. - The barred spirals UDF 1971 (left) and UDF 9341 are shown with their radial distributions of position angle and ellipticity. 


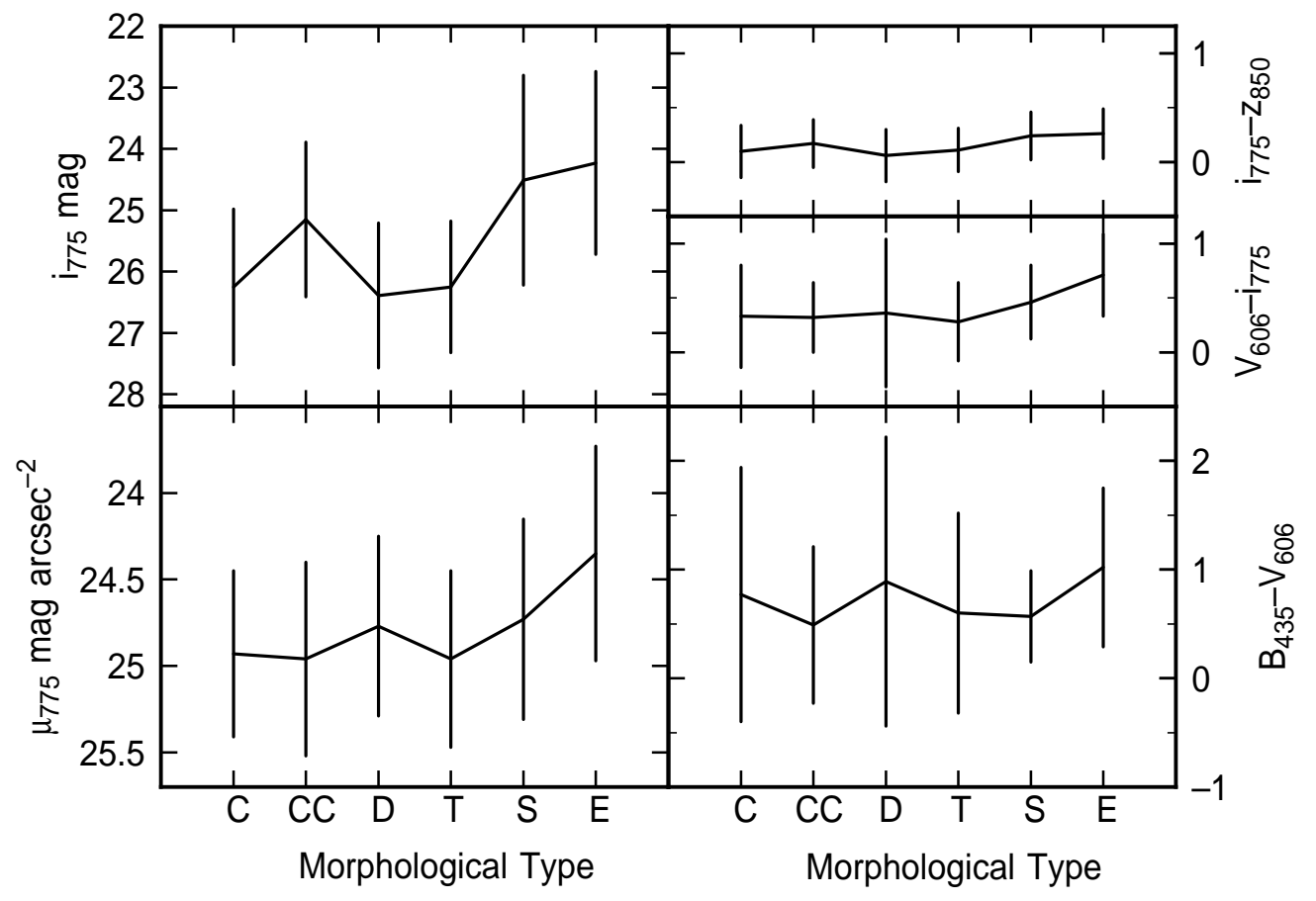

Fig. 4.- The average magnitudes (upper left), surface brightnesses (lower left), and colors (right) are shown for each galaxy type, with $1 \sigma$ rms fluctuations from sample varieties. Ellipticals are the reddest and brightest galaxies. 


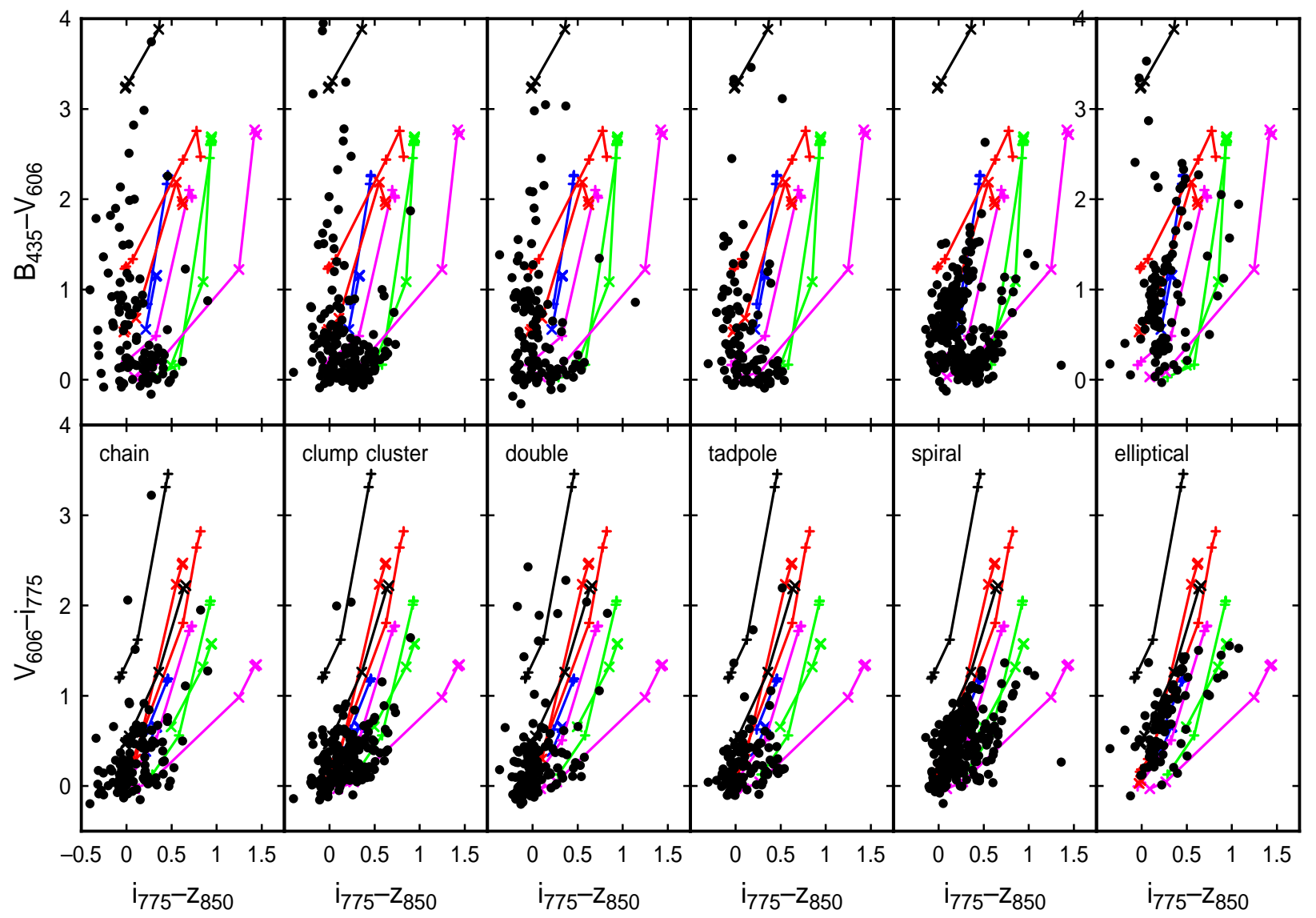

Fig. 5.- Color-color distributions are shown for all 884 galaxies divided by morphology. Superposed curves are stellar evolution models for redshifts 0 (blue x symbol), 0.5 (blue $+), 1($ green $\mathrm{x}), 1.5($ green +$), 2($ magenta $\mathrm{x}), 2.5($ magenta +$), 3(\operatorname{red} \mathrm{x}), 3.5($ red +$), 4$ (black $\mathrm{x}$ ), and 4.5 (black + ). The models assume star formation began at $z=6$ and decayed exponentially; the curves trace out the colors as the e-folding time in Gy varies as 0.01, 0.03, $0.1,0.3,1,3$, and infinity (high decay times are the most blue). 

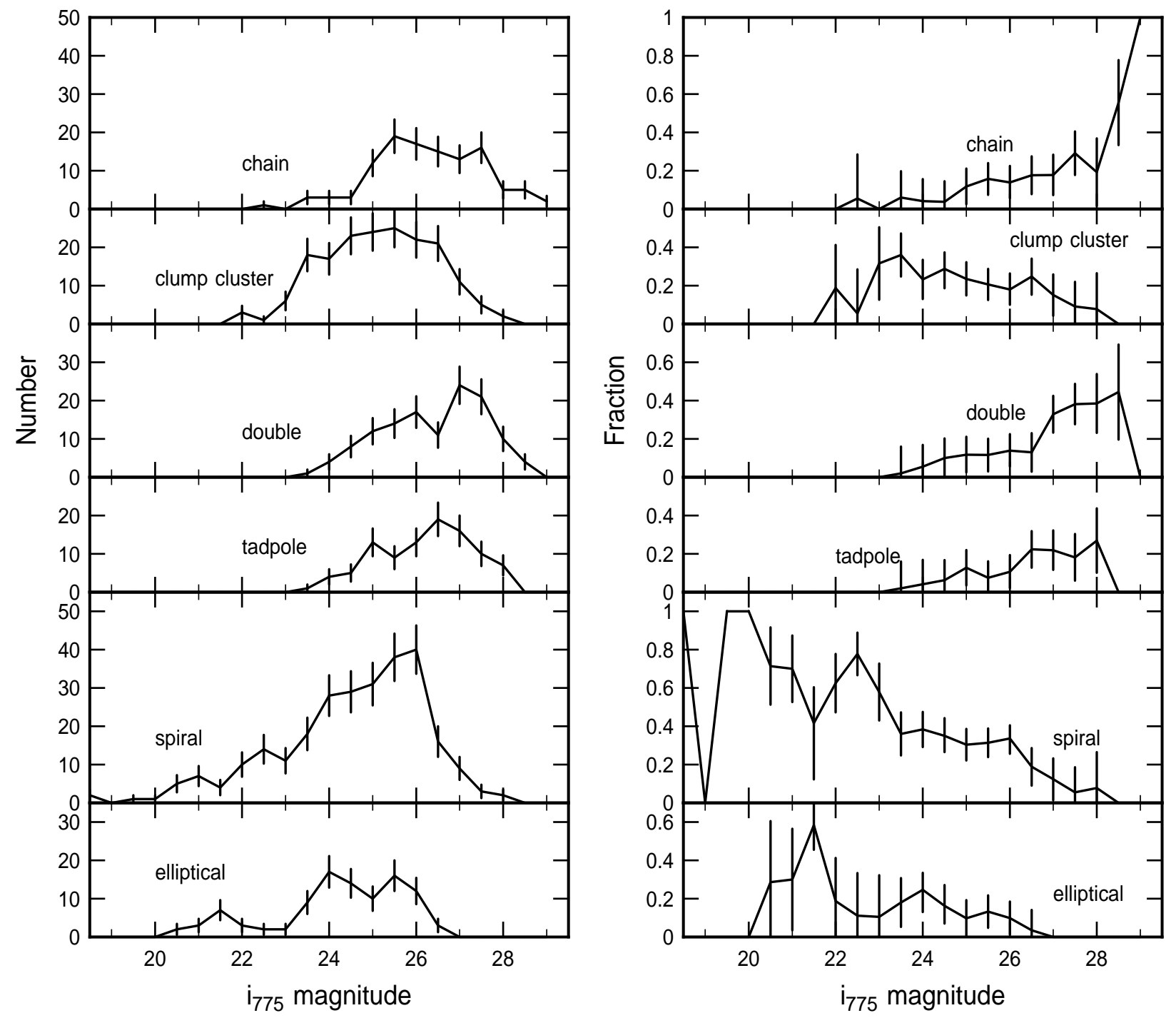

Fig. 6. - The number and fraction of each galaxy type is shown as a function of apparent magnitude, with $1 \sigma$ error bars. Linear structures dominate at the faintest magnitudes. 


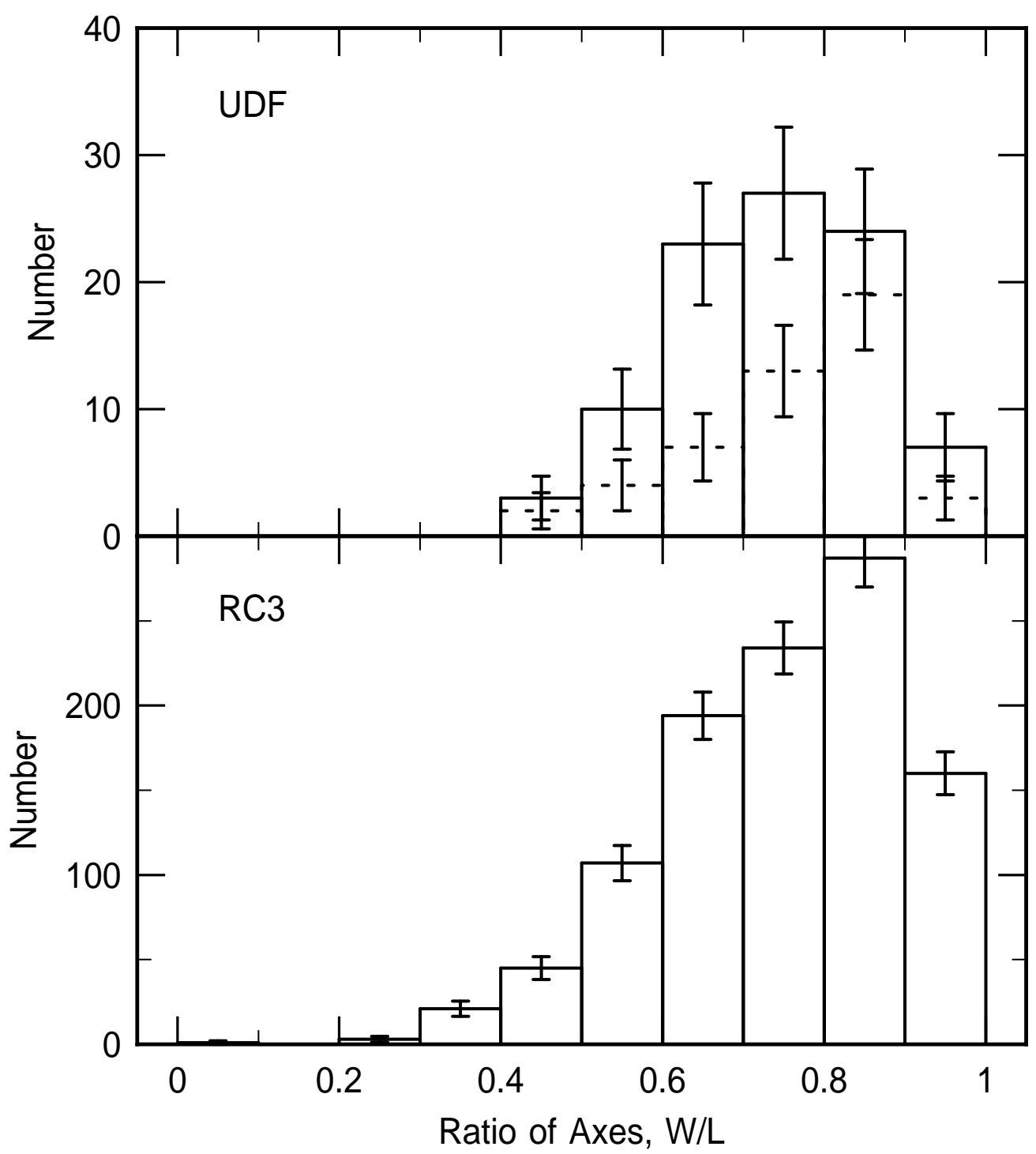

Fig. 7.- The distribution of the ratio of axis for elliptical galaxies in the UDF compared to the RC3. The dotted histogram for the UDF is for the half with the highest surface brightness. The UDF distribution resembles the local distribution, suggesting that elliptical galaxy shapes are standard and were probably determined relatively quickly in the early Universe. 


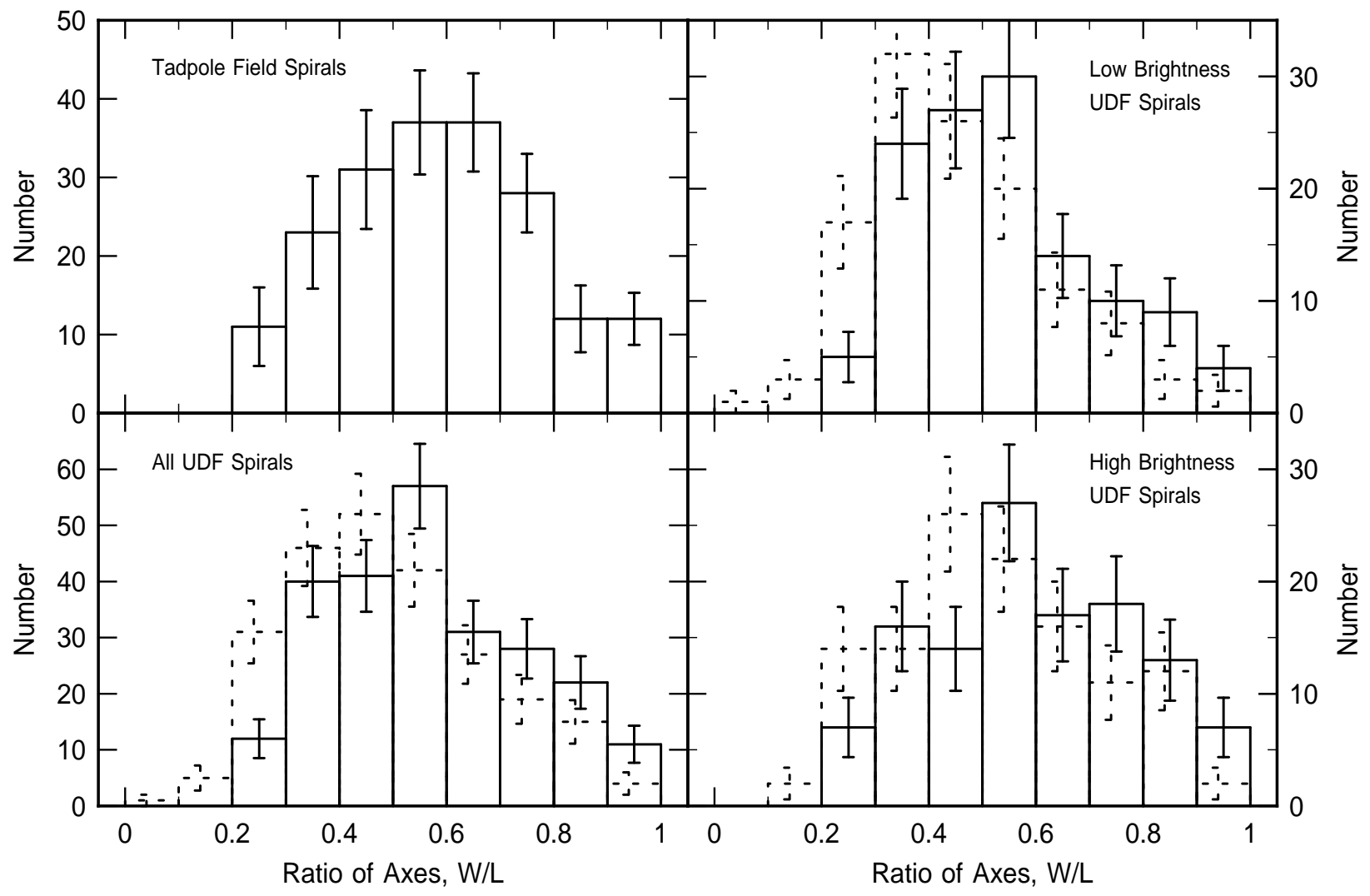

Fig. 8. - The distribution of the ratio of axis (width to length, $W / L$ ) for spiral galaxies in the deep field of the Tadpole Galaxy and in the UDF field. The distributions differ significantly from the local distributions (Fig. 9) because of a lack of face-on spirals at high redshift. Randomly oriented disks would have a flat distribution in a diagram like this. The fall-off at low $W / L$ results from the intrinsic disk thickness. The position of this fall-off is significantly larger at high redshift than for local galaxies, indicating that the high redshift galaxies have thicker disks. 


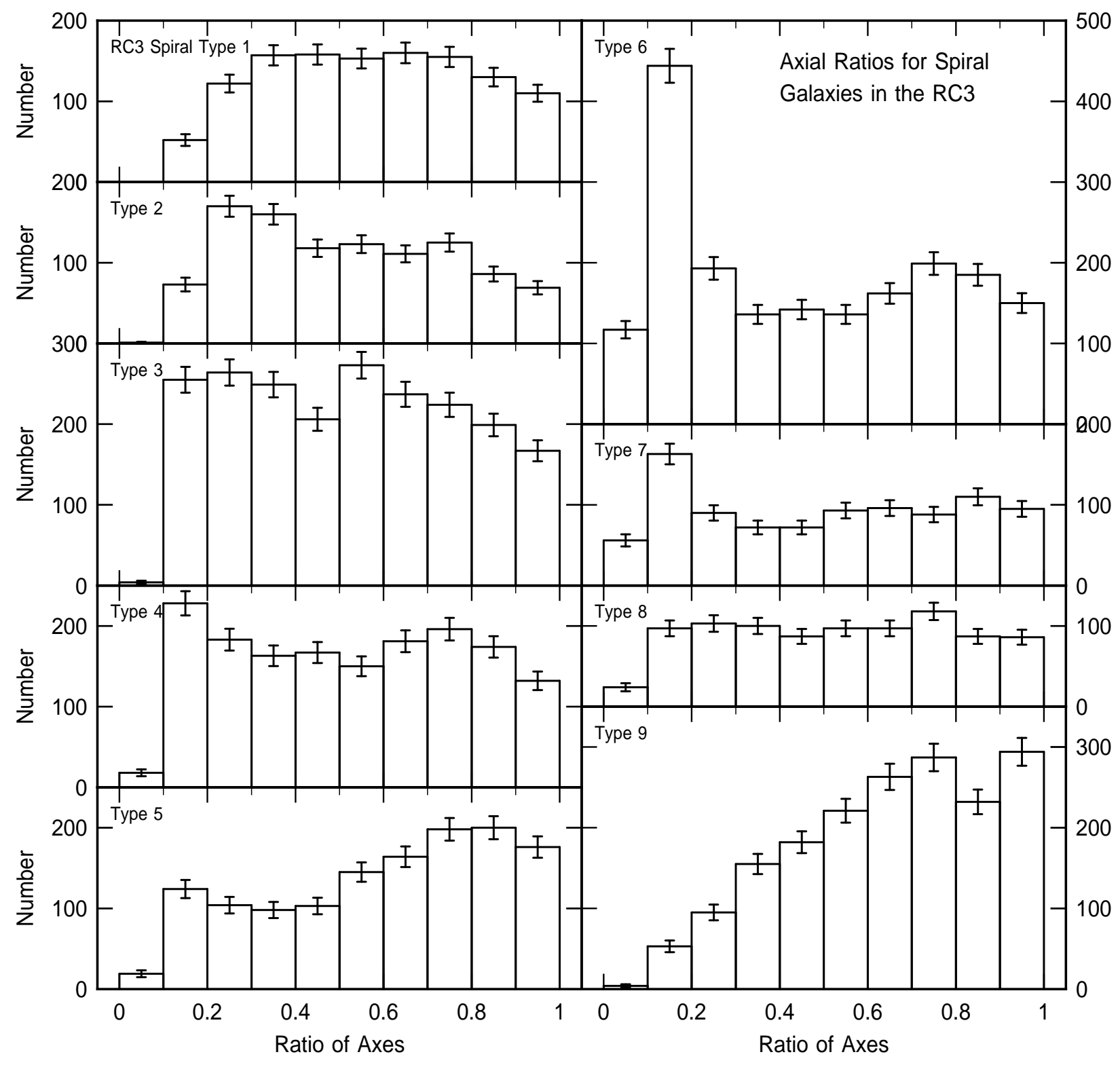

Fig. 9.- The distribution of the ratio of axis for spiral galaxies in the RC3, divided according to de Vaucouleurs type. 


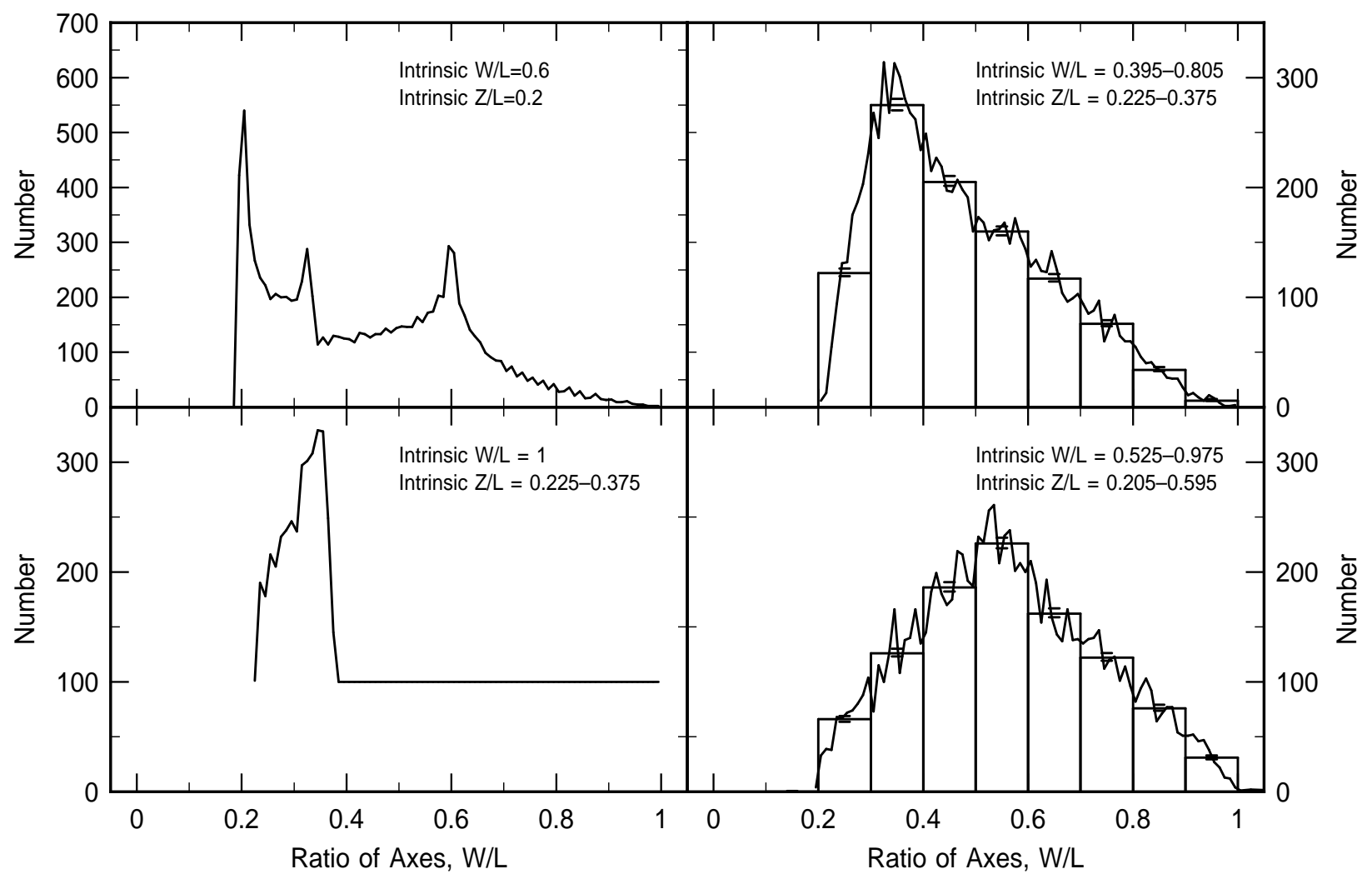

Fig. 10.- Models of distribution of axial ratio for intrinsically triaxial galaxies viewed at random angles, demonstrating how the observed deviations from a uniform distribution could arise at high redshift without surface brightness selection effects. The range of intrinsic width to length $(W / L)$ and thickness to length $(Z / L)$ is indicated on each panel. The two distributions on the right illustrate how the centrally peaked and skewed distributions of the observed spirals can be matched by a triaxial model. 


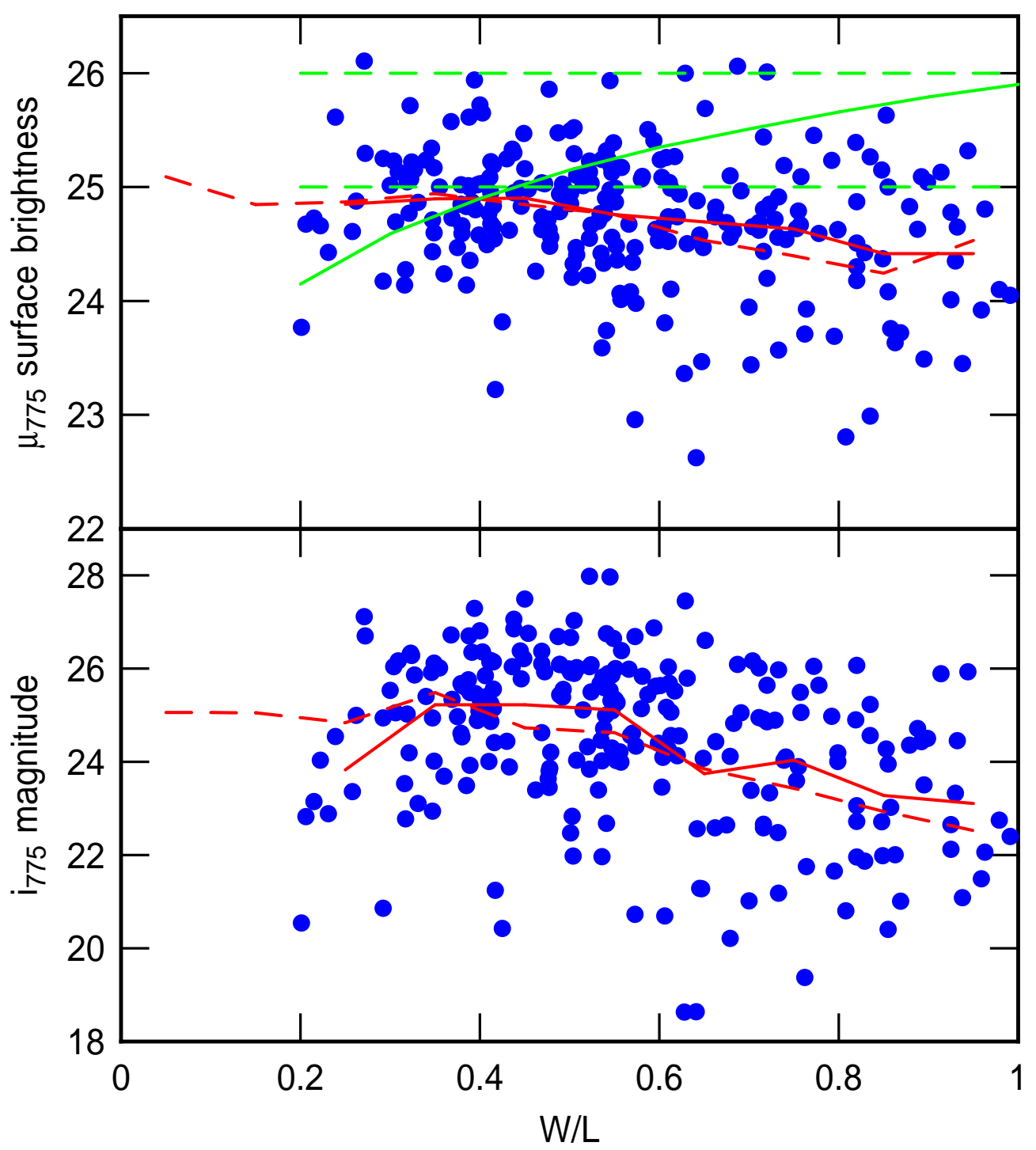

Fig. 11.- The distribution of magnitude and surface brightness for all of the spiral galaxies in this catalog, plotted versus the apparent ratio of axes, $W / L$. The plotted points and the solid red lines use the axial ratios tabulated in the on-line UDF catalog, while the dashed red lines use the ratio of axes determined by the authors from individual ellipse fits on IRAF. In the bottom panel. the edge-on galaxies are fainter as a result of their lower projected areas. In the top panel, the dashed green lines represent the range of surface brightnesses where galaxies begin to drop below the detection limit. The solid green line is a model where the surface brightness is proportional to the path length through the disk. As the path length decreases for more face-on systems, and the average surface brightness becomes fainter, the detection limit is passed and the galaxies disappear from view, making the density of plotted points lower. 


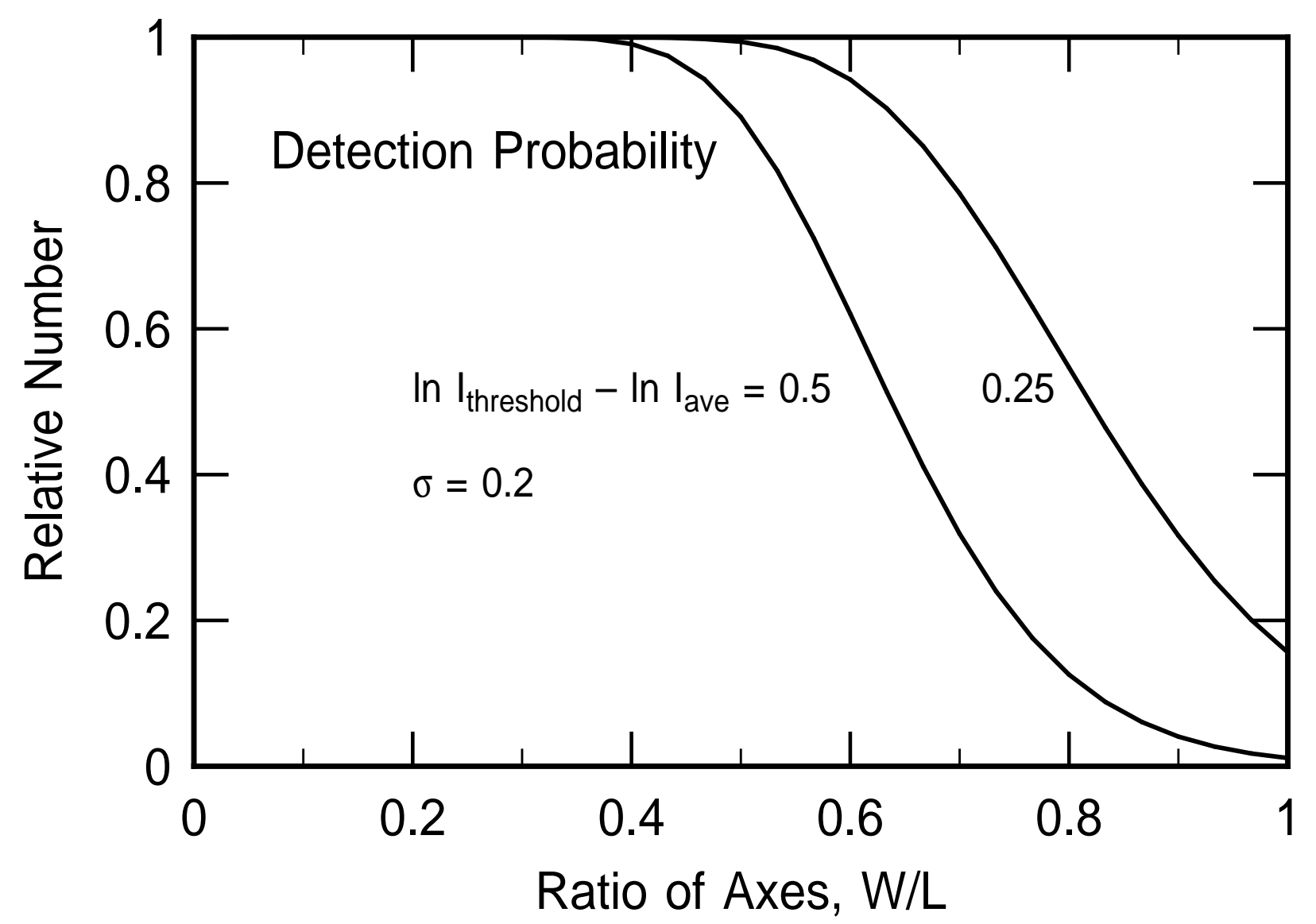

Fig. 12.- A model for the detection probability of spirals which have a distribution of faceon surface brightnesses that is Gaussian in magnitude (i.e., log-normal in intensity) with a dimensionless dispersion $\sigma=0.2$ and a value at the peak, $I_{\text {ave }}$, fainter than the survey threshold, $I_{\text {threshold }}$ by factors of $e^{0.5}$ and $e^{0.25}$. These two cases bracket the observed fall-off in galaxy counts for the range of $W / L$ between 0.5 and 1 (as in Fig. 8). 